\title{
RELAXATION TIMES FOR ATOM DISLOCATIONS IN CRYSTALS
}

\author{
STEFANIA PATRIZI AND ENRICO VALDINOCI
}

\begin{abstract}
We study the relaxation times for a parabolic differential equation whose solution represents the atom dislocation in a crystal. The equation that we consider comprises the classical Peierls-Nabarro model as a particular case, and it allows also long range interactions.

It is known that the dislocation function of such a model has the tendency to concentrate at single points, which evolve in time according to the external stress and a singular, long range potential.

Depending on the orientation of the dislocation function at these points, the potential may be either attractive or repulsive, hence collisions may occur in the latter case and, at the collision time, the dislocation function does not disappear.

The goal of this paper is to provide accurate estimates on the relaxation times of the system after collision. More precisely, we take into account the case of two and three colliding points, and we show that, after a small transition time subsequent to the collision, the dislocation function relaxes exponentially fast to a steady state.

In this sense, the system exhibits two different decay behaviors, namely an exponential time decay versus a polynomial decay in the space variables (and these two homogeneities are kept separate during the time evolution).
\end{abstract}

\section{INTRODUCTION}

In this paper we consider a function $v_{\varepsilon}(t, x)$, which depends on the time variable $t \geqslant 0$ and the space variable $x \in \mathbb{R}$, and which represents the atom dislocation in a crystal (in this setting, the small parameter $\varepsilon>0$ represents the size of the periodicity of the crystal).

The evolution of $v_{\varepsilon}(t, x)$ is governed by a parabolic equation of nonlocal type, in which the variation of $v_{\varepsilon}$ in time is produced by an elastic, or ferromagnetic, effect and is influenced by the periodic structure of the crystal at a large scale. These types of equations have been widely studied after the pioneer work of Peierls and Nabarro (see e.g. [11, 8] and the references therein). Moreover, some generalizations of the original model of Peierls and Nabarro have been recently considered to take into account long range interactions with different scales (see [4, 3]) and the system can also be linked to the classical model at the atomic scale which was introduced by Frenkel and Kontorova (see [6]). Different space/time scale of the model also produce homogenization results, whose effective Hamiltonian depends on the scaling properties of the operator (in particular, this Hamiltonian may present either local or nonlocal features, see [10, 15]). We also refer to [9, 12] for some parabolic equations with classical diffusion and multiple-well potentials.

2010 Mathematics Subject Classification. 82D25, 35R09, 74E15, 35R11, 47G20.

Key words and phrases. Peierls-Nabarro model, nonlocal integro-differential equations, dislocation dynamics, attractive/repulsive potentials, collisions.

The authors have been supported by the ERC grant 277749 "EPSILON Elliptic Pde's and Symmetry of Interfaces and Layers for Odd Nonlinearities". 
For small $\varepsilon$, the dislocation function $v_{\varepsilon}$ approaches a piecewise constant function (see [8, [4, 3, 14]). The plateaus of this asymptotic limit correspond to the periodic sites induced by the crystalline structure, but its jump points evolve in time, according to the external stress and a singular potential. Roughly speaking, one can imagine that the discontinuity points of this limit dislocation function behave like a "particle" system (though no "material" particle is really involved), driven by a system of ordinary differential equations which describe the position of the jump points $x_{1}(t), \ldots, x_{N}(t)$.

We refer to Section 2 in [4] for a discussion of the link between the integro-differential equation which governs the evolution of the dislocation function $v_{\varepsilon}$ and the system of ODE's which drives the particles $x_{1}, \ldots, x_{N}$. See in particular Subsection 2.2 of [14] for a detailed heuristic discussion. Remarkably, the physical properties of the singular potential of this ODE system depend on the orientation of the dislocation at the jump points. Namely, if the dislocation function has the same spatial monotonicity at $x_{i}$ and $x_{i+1}$, then the potential induces a repulsion between the particles $x_{i}$ and $x_{i+1}$. Conversely, when the dislocation function has opposite spatial monotonicity at $x_{i}$ and $x_{i+1}$, then the potential becomes attractive, and the two particles may collide in a finite time $T_{c}$. In formulas, in the collision case we have that $x_{i}(t) \neq x_{i+1}(t)$ for any $t \in\left[0, T_{c}\right)$, with

$$
\lim _{t \rightarrow T_{c}^{-}} x_{i}\left(T_{c}\right)=\lim _{t \rightarrow T_{c}^{-}} x_{i+1}\left(T_{c}\right)=: x_{c} .
$$

Often, we will use the notation $x_{i}\left(T_{c}\right)=x_{i+1}\left(T_{c}\right)$ to denote the collision described by (1.1).

At the collision time $T_{c}$, the dislocation function does not get annihilated. More precisely, it asymptotically vanishes outside the collision point $x_{c}$, but, in general,

$$
\limsup _{\substack{t \rightarrow T_{c}^{-} \\ \varepsilon \rightarrow 0^{+}}} v_{\varepsilon}\left(t, x_{c}\right) \geqslant 1
$$

Roughly speaking, this suggests that the dislocation function keeps some nontrivial effect after the collision time (notice indeed that, since the jump points $x_{i}$ do not correspond to a "material" particle, the evolution of the dislocation function $v_{\varepsilon}$ persists even after the collision time $T_{c}$ ).

The objective of this paper is therefore to study the behavior of the dislocation function after the collision time $T_{c}$. We will prove that there exists a transition time $T_{\varepsilon}$ (with $T_{\varepsilon}>$ $T_{c}$, and $T_{\varepsilon} \rightarrow T_{c}$ as $\varepsilon \rightarrow 0^{+}$) such that, when $t \geqslant T_{\varepsilon}$, the dislocation function decays to the steady state exponentially fast in time, uniformly with respect to the space variable.

More precisely, we will consider here the case of two and three particles and show that the limit configuration of $v_{\varepsilon}$ is either a constant (in the case of two particles) or a heteroclinic (in the case of three particles). We show that at the time $T_{\varepsilon}$ the dislocation function $v_{\varepsilon}$ gets close to this limit configuration, and, for $t \geqslant T_{\varepsilon}$, the dislocation approaches the limit exponentially fast.

This exponential decay may be explicitly quantified via the expression

$$
e^{c \frac{T_{\varepsilon}-t}{\varepsilon^{2 s+1}}}
$$

where $c$ is a positive constant and $2 s \in(0,2)$ is the order of the integro-differential operator in the evolution equation (the case $s=1 / 2$ has indeed special physical interest, see e.g. $[11,8])$. It is worth to point out that the decay in (1.2) improves as $\varepsilon \rightarrow 0^{+}$. 
We also stress that such exponential decay is not obvious from the beginning. On the contrary, solutions of integro-differential equations in general present a polynomial (and not an exponential) tail, see e.g. [13], and also in our case the transitions considered have only a polynomial decay in the space variables. In a sense, the exponential decay in (1.2) is a consequence of the fact that, at the right space/time scale, the integro-differential operator acts only in the space coordinates, allowing the time derivative (which is a local operator) to recover the exponential decay of classical flavor.

We stress that the results of the present paper are new even in the case $s=1 / 2$.

For the formal mathematical treatment of this model, we introduce the following notation. We consider the problem

$$
\begin{cases}\left(v_{\varepsilon}\right)_{t}=\frac{1}{\varepsilon}\left(\mathcal{I}_{s} v_{\varepsilon}-\frac{1}{\varepsilon^{2 s}} W^{\prime}\left(v_{\varepsilon}\right)+\sigma(t, x)\right) & \text { in }(0,+\infty) \times \mathbb{R} \\ v_{\varepsilon}(0, \cdot)=v_{\varepsilon}^{0} & \text { on } \mathbb{R}\end{cases}
$$

where $\varepsilon>0$ is a small scale parameter, $W$ is a periodic potential and $\mathcal{I}_{s}$ is the so-called fractional Laplacian of any order $2 s \in(0,2)$. Precisely, given $\varphi \in C^{2}\left(\mathbb{R}^{N}\right) \cap L^{\infty}\left(\mathbb{R}^{N}\right)$, let us define

$$
\mathcal{I}_{s}[\varphi](x):=P V \int_{\mathbb{R}^{N}} \frac{\varphi(x+y)-\varphi(x)}{|y|^{N+2 s}} d y
$$

where $P V$ stands for the principal value of the integral. We refer to [17] and [5] for a basic introduction to the fractional Laplace operator. On the potential $W$ we assume

$$
\begin{cases}W \in C^{3, \alpha}(\mathbb{R}) & \text { for some } 0<\alpha<1 \\ W(v+1)=W(v) & \text { for any } v \in \mathbb{R} \\ W=0 & \text { on } \mathbb{Z} \\ W>0 & \text { on } \mathbb{R} \backslash \mathbb{Z} \\ W^{\prime \prime}(0)>0 . & \end{cases}
$$

The function $\sigma$ satisfies:

$$
\left\{\begin{array}{l}
\sigma \in B U C([0,+\infty) \times \mathbb{R}) \quad \text { and for some } M>0 \text { and } \alpha \in(s, 1) \\
\left\|\sigma_{x}\right\|_{L^{\infty}([0,+\infty) \times \mathbb{R})}+\left\|\sigma_{t}\right\|_{L^{\infty}([0,+\infty) \times \mathbb{R})} \leqslant M \\
\left|\sigma_{x}(t, x+h)-\sigma_{x}(t, x)\right| \leqslant M|h|^{\alpha}, \quad \text { for every } x, h \in \mathbb{R} \text { and } t \in[0,+\infty) .
\end{array}\right.
$$

From the viewpoint of physics, $W$ represents the potential produced by the periodicity of the crystal at a large scale and $\sigma$ is an external forcing term (see [4] for a more detailed discussion).

In this paper we consider the case in which the initial condition in $(1.3)$ is a superposition of either two or three transition layers with different orientation. Precisely, let us introduce the so-called basic layer solution $u$ associated to $\mathcal{I}_{s}$, that is the solution of

$$
\left\{\begin{array}{lr}
\mathcal{I}_{s}(u)=W^{\prime}(u) & \text { in } \mathbb{R} \\
u^{\prime}>0 & \text { in } \mathbb{R} \\
\lim _{x \rightarrow-\infty} u(x)=0, \quad \lim _{x \rightarrow+\infty} u(x)=1, & u(0)=\frac{1}{2} .
\end{array}\right.
$$


This is the basic transition layer that we will use to construct our initial data. Namely, we will consider in this paper two types of initial data. The first case deals with the superposition of two transition layers with opposite orientations: in this case the points associated with the transitions attract each other, a collision occurs and slightly after collision the system goes to rest exponentially fast. The second situation considers three transition layers with alternate orientations: in this case, the middle point is attractive for the two external ones, a collision (possibly, a multiple collision) occurs and after a short transient time the system approaches exponentially fast the steady state given by a single transition layer.

These results will be rigorously presented in the forthcoming Subsections 1.1 and 1.2 .

1.1. The case of two transition layers. Given $x_{1}^{0}<x_{2}^{0}$ let us consider as initial condition in 1.3

$$
v_{\varepsilon}^{0}(x)=\frac{\varepsilon^{2 s}}{\beta} \sigma(0, x)+u\left(\frac{x-x_{1}^{0}}{\varepsilon}\right)+u\left(\frac{x_{2}^{0}-x}{\varepsilon}\right)-1,
$$

where

$$
\beta:=W^{\prime \prime}(0)>0,
$$

and $u$ is solution of (1.7). One may consider in the formula above the term $\sigma(0, x)$ as the effect on the dislocation of the external stess at the initial time (of course, if no stress is applied at the initial time, this additional dislocation vanishes). Let us introduce the solution $\left(x_{1}(t), x_{2}(t)\right)$ to the system

$$
\begin{cases}\dot{x}_{1}=\gamma\left(-\frac{x_{1}-x_{2}}{2 s\left|x_{1}-x_{2}\right|^{2 s+1}}-\sigma\left(t, x_{1}\right)\right) & \text { in }\left(0, T_{c}\right) \\ \dot{x}_{2}=\gamma\left(-\frac{x_{2}-x_{1}}{2 s\left|x_{2}-x_{1}\right|^{2 s}}+\sigma\left(t, x_{2}\right)\right) & \text { in }\left(0, T_{c}\right) \\ x_{1}(0)=x_{1}^{0}, x_{2}(0)=x_{2}^{0} & \end{cases}
$$

where

$$
\gamma:=\left(\int_{\mathbb{R}}\left(u^{\prime}(x)\right)^{2} d x\right)^{-1}
$$

and $\left(0, T_{c}\right)$ is the maximal interval where the system 1.10$)$ is well defined, i.e. $x_{1}(t)<$ $x_{2}(t)$ for any $t \in\left[0, T_{c}\right)$ and $x_{1}\left(T_{c}\right)=x_{2}\left(T_{c}\right)$.

In general, it may happen that $T_{c}=+\infty$, i.e. no collision occurs. On the other hand, it can be shown that when either the external stress is small or the particles are initially close to collision, then $T_{c}<+\infty$. More precisely, in [15] we proved that if the following condition is satisfied

$$
\text { either } \sigma \leqslant 0 \quad \text { or } \quad x_{2}^{0}-x_{1}^{0}<\left(\frac{1}{2 s\|\sigma\|_{\infty}}\right)^{\frac{1}{2 s}},
$$

then the collision time $T_{c}$ is finite.

In the setting of finite collision time, we prove here that the dislocation function $v_{\varepsilon}$, after a time $T_{\varepsilon}$, which is only slightly larger than the collision time $T_{c}$, becomes small with $\varepsilon$. The precise result goes as follows: 
Theorem 1.1. Assume that (1.5), (1.6), (1.8) hold and $T_{c}<+\infty$. Let $v_{\varepsilon}$ be the solution of (1.3)-(1.8). Then there exists $\varepsilon_{0}>0$ such that for any $\varepsilon<\varepsilon_{0}$ there exist $T_{\varepsilon}, \varrho_{\varepsilon}>0$ such that

$$
T_{\varepsilon}=T_{c}+o(1), \quad \varrho_{\varepsilon}=o(1) \quad \text { as } \varepsilon \rightarrow 0
$$

and

$$
v_{\varepsilon}\left(T_{\varepsilon}, x\right) \leqslant \varrho_{\varepsilon} \quad \text { for any } x \in \mathbb{R} .
$$

The result above can be made precise by saying that, if the system is not subject to any external stress, then the dislocation function $v_{\varepsilon}$ decays in time exponentially fast. More precisely, we have:

Theorem 1.2. Assume that (1.5), (1.6), (1.8) hold and that $\sigma \equiv 0$. Let $v_{\varepsilon}$ be the solution of (1.3)-(1.8). Then there exist $\varepsilon_{0}>0$ and $c>0$ such that for any $\varepsilon<\varepsilon_{0}$ we have

$$
\left|v_{\varepsilon}(t, x)\right| \leqslant \varrho_{\varepsilon} e^{c \frac{T_{\varepsilon}-t}{\varepsilon^{2 s+1}}}, \quad \text { for any } x \in \mathbb{R} \text { and } t \geqslant T_{\varepsilon},
$$

where $T_{\varepsilon}$ and $\varrho_{\varepsilon}$ are given in Theorem 1.1 .

The evolution of the two particle system and of the associated dislocation function, as obtained in Theorems 1.1 and 1.2, is described in Figure 1.

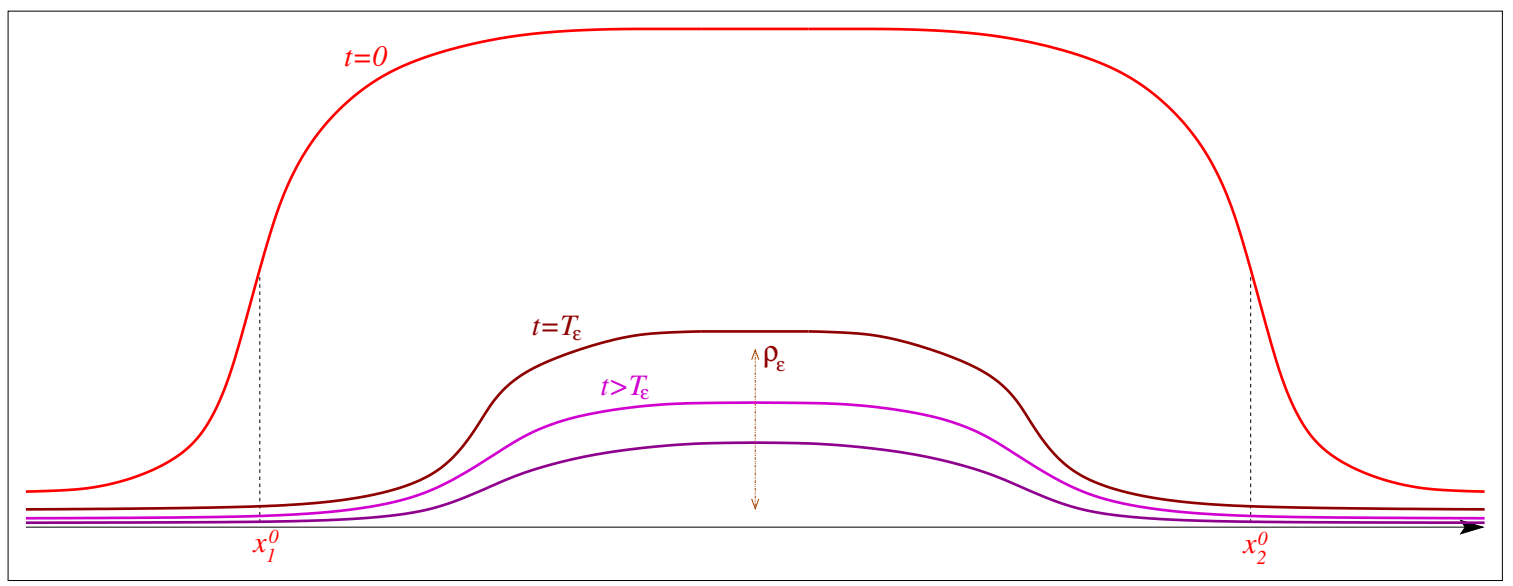

Figure 1: Evolution of the dislocation function in case of two particles.

1.2. The case of three transition layers. Next, we consider the case in which the initial condition in $(1.3)$ is a superposition of three transition layers with different orientation. Precisely, let $\zeta_{1}=1, \zeta_{2}=-1, \zeta_{3}=1$. Given $x_{1}^{0}<x_{2}^{0}<x_{3}^{0}$, let us consider as initial condition in 1.3

$$
v_{\varepsilon}^{0}(x)=\frac{\varepsilon^{2 s}}{\beta} \sigma(0, x)+\sum_{i=1}^{3} u\left(\zeta_{i} \frac{x-x_{i}^{0}}{\varepsilon}\right)-1,
$$


where $\beta$ is given by (1.9) and $u$ is solution of (1.7). Let us introduce the solution $\left(x_{1}(t), x_{2}(t), x_{3}(t)\right)$ to the following system: for $i=1,2,3$

$$
\left\{\begin{array}{l}
\dot{x}_{i}=\gamma\left(\sum_{j \neq i} \zeta_{i} \zeta_{j} \frac{x_{i}-x_{j}}{2 s\left|x_{i}-x_{j}\right|^{1+2 s}}-\zeta_{i} \sigma\left(t, x_{i}\right)\right) \quad \text { in }\left(0, T_{c}\right) \\
x_{i}(0)=x_{i}^{0}
\end{array}\right.
$$

where $\gamma$ is given by 1.11 and $T_{c}$ is the collision time of system (1.15), i.e.

$$
x_{i+1}(t)>x_{i}(t) \text { for any } t \in\left[0, T_{c}\right) \text { and } i=1,2
$$

and there exist $i_{0}$ such that

$$
x_{i_{0}+1}\left(T_{c}\right)=x_{i_{0}}\left(T_{c}\right) .
$$

The first result that we prove in the three particle case is the analogue of Theorem 1.1 . That is, we show that after some time that is just slightly bigger than the collision time, the dislocation function becomes comparable, up to a small error, with the associated steady state. The case of three particles is, on the other hand, different from the case of two particles, since the steady state associated with the case of three particles is the heteroclinic (and not the trivial function as in the case of two particles).

This phenomenon may be, roughly speaking, explained by the fact that in case of two particles, the collision of the two particles "annihilate" all the dynamics, nothing more is left and the system relaxes to the trivial equilibrium. Conversely, in the case of three particles, one has that two particles "annihilate" each other, but the third particle "survives", and this produces a jump in the dislocation function - indeed, these "purely mathematical" particles correspond to an excursion of the dislocation, from two equilibria, which is modeled by the standard transition layer in (1.7). The precise result is the following:

Theorem 1.3. Assume that (1.5), (1.6), (1.8) hold and $T_{c}<+\infty$. Let $v_{\varepsilon}$ be the solution of (1.3) -(1.14). Then there exists $\varepsilon_{0}>0$ such that for any $\varepsilon<\varepsilon_{0}$ there exist $T_{\varepsilon}^{1}, T_{\varepsilon}^{2}, \varrho_{\varepsilon}>0$ and $y_{\varepsilon}, z_{\varepsilon}$ such that

$$
\begin{gathered}
T_{\varepsilon}^{1}, T_{\varepsilon}^{2}=T_{c}+o(1), \quad \varrho_{\varepsilon}=o(1) \quad \text { as } \varepsilon \rightarrow 0, \\
\left|z_{\varepsilon}-y_{\varepsilon}\right|=o(1) \quad \text { as } \varepsilon \rightarrow 0
\end{gathered}
$$

and for any $x \in \mathbb{R}$

$$
v_{\varepsilon}\left(T_{\varepsilon}^{1}, x\right) \leqslant u\left(\frac{x-y_{\varepsilon}}{\varepsilon}\right)+\varrho_{\varepsilon}
$$

and

$$
v_{\varepsilon}\left(T_{\varepsilon}^{2}, x\right) \geqslant u\left(\frac{x-z_{\varepsilon}}{\varepsilon}\right)-\varrho_{\varepsilon},
$$

where $u$ is the solution of 1.7 .

Next result is the analogue of Theorem 1.2 in the three particle setting. Roughly speaking, it says that, after a small transition time after the collision, the dislocation function relaxes towards the standard layer solution exponentially fast. The formal statement is the following: 
Theorem 1.4. Assume that (1.5), (1.6), (1.8) hold and that $\sigma \equiv 0$. Let $v_{\varepsilon}$ be the solution of (1.3)-(1.14). Then there exist $\varepsilon_{0}>0$ and $\mu>0$ such that for any $\varepsilon<\varepsilon_{0}$ there exists $K_{\varepsilon}=o(1)$ as $\varepsilon \rightarrow 0$ such that

$v_{\varepsilon}(t, x) \leqslant u\left(\frac{x-y_{\varepsilon}+K_{\varepsilon} \varrho_{\varepsilon}\left(1-e^{-\frac{\mu\left(t-T_{\varepsilon}^{1}\right)}{\varepsilon^{2 s+1}}}\right)}{\varepsilon}\right)+\varrho_{\varepsilon} e^{-\frac{\mu\left(t-T_{\varepsilon}^{1}\right)}{\varepsilon^{2 s+1}}}, \quad$ for any $x \in \mathbb{R}$ and $t \geqslant T_{\varepsilon}^{1}$,

$v_{\varepsilon}(t, x) \geqslant u\left(\frac{x-z_{\varepsilon}-K_{\varepsilon} \varrho_{\varepsilon}\left(1-e^{-\frac{\mu\left(t-T_{\varepsilon}^{2}\right)}{\varepsilon^{2 s+1}}}\right)}{\varepsilon}\right)-\varrho_{\varepsilon} e^{-\frac{\mu\left(t-T_{\varepsilon}^{2}\right)}{\varepsilon^{2 s+1}}}, \quad$ for any $x \in \mathbb{R}$ and $t \geqslant T_{\varepsilon}^{2}$

where $T_{\varepsilon}^{1}, T_{\varepsilon}^{2}, \varrho_{\varepsilon}, y_{\varepsilon}$ and $z_{\varepsilon}$ are given in Theorem 1.3 and $u$ is the solution of (1.7).

Corollary 1.5. Under the assumptions of Theorem 1.4, there exists $\varepsilon_{0}>0$ such that for any $\varepsilon<\varepsilon_{0}$, there exist a sequence $t_{k} \rightarrow+\infty$ as $k \rightarrow+\infty$, and a point $x_{\varepsilon} \in \mathbb{R}$ with

$$
y_{\varepsilon}-K_{\varepsilon} \varrho_{\varepsilon}<x_{\varepsilon}<z_{\varepsilon}+K_{\varepsilon} \varrho_{\varepsilon},
$$

such that

$$
v_{\varepsilon}\left(t_{k}, x\right) \rightarrow u\left(\frac{x-x_{\varepsilon}}{\varepsilon}\right) \quad \text { as } k \rightarrow+\infty,
$$

where $y_{\varepsilon}, z_{\varepsilon}, K_{\varepsilon}$ and $\varrho_{\varepsilon}$ are given in Theorem 1.3 and $u$ is the solution of (1.7).

The results of Theorems 1.3 and 1.4 and Corollary 1.5 are represented in Figure 2, where we sketched the evolution of the dislocation function and of the associated particle system in the case of three particles with alternate orientations.

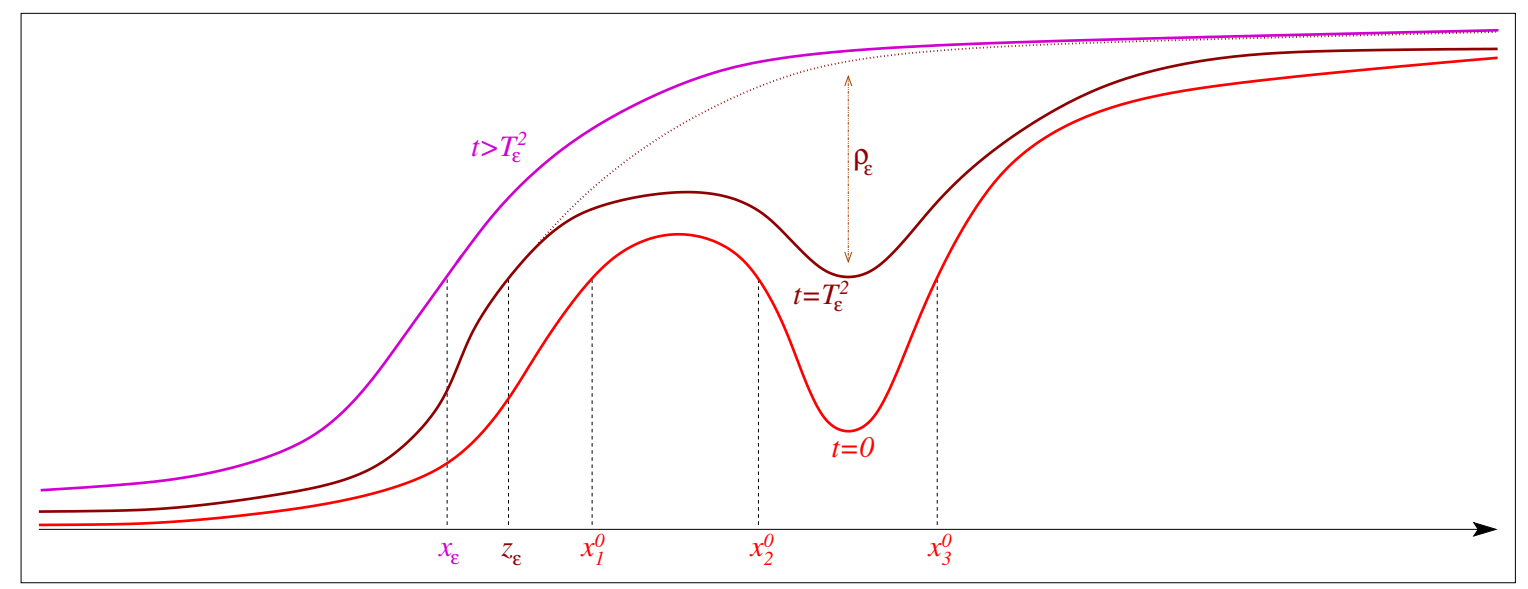

Figure 2: Evolution of the dislocation function in case of three particles.

Notice that the external stress $\sigma$ is of course given and does not depend on the orientation of the dislocation, since it is an external force. Nevertheless its elastic effect on the 
motion of the dislocations do depend on the relative orientations, as given in $(1.10)$ and (1.15).

It is worth to point out that the case of three particles provides structurally richer phenomena than the case of two particles. Indeed, in the case of three particles we have two different types of collision: simple and triple. The simple collision occurs when only two particles collide at time $T_{c}$, i.e., either

$$
x_{1}\left(T_{c}\right)=x_{2}\left(T_{c}\right) \quad \text { and } \quad x_{3}\left(T_{c}\right)>x_{2}\left(T_{c}\right),
$$

or

$$
x_{2}\left(T_{c}\right)=x_{3}\left(T_{c}\right) \quad \text { and } \quad x_{1}\left(T_{c}\right)<x_{2}\left(T_{c}\right) .
$$

In the triple collision case, the three particles collide together and simultaneously, i.e.

$$
x_{1}\left(T_{c}\right)=x_{2}\left(T_{c}\right)=x_{3}\left(T_{c}\right) .
$$

In [14], we proved that if $\sigma \equiv 0$, then for any choice of the initial condition $\left(x_{1}^{0}, x_{2}^{0}, x_{3}^{0}\right)$ we have a collision in a finite time. Moreover a triple collision is possible if and only if

$$
x_{2}^{0}-x_{1}^{0}=x_{3}^{0}-x_{2}^{0} .
$$

The proofs of the results in the three particle setting will have to take into account the distinction between simple and triple collisions (on the one hand, the simple collision is "more generic" and less singular, on the other hand, the triple collision case has the technical advantage of concentrating all the relevant phenomena of the dynamics at just a single point).

Additional results concerning relaxation times and asymptotics of the Peierls-Nabarro model will be given in the forthcoming paper [16].

The rest of the paper is organized as follows. In Section 2 we discuss the basic properties of the basic transition layer and of the solution of a corrector equation. The main results of this paper (that are Theorems 1.1, 1.2, 1.3 and 1.4, and Corollary 1.5) are proved in Sections 3, 4, 5, 6 and 7.

The proof of the main results rely on some auxiliary lemmata which can be proved simultaneously in the case of two particles and in the case of three particles: for this reason, the proof of all these common results is postponed to Section 8 .

\section{Preliminary observations}

2.1. Toolbox. In this section we recall some general auxiliary results that will be used in the rest of the paper. In what follows we denote by $H$ the Heaviside function.

Lemma 2.1. Assume that (1.5) holds, then there exists a unique solution $u \in C^{2, \alpha}(\mathbb{R})$ of (1.7). Moreover, there exist constants $C, c>0$ and $\kappa>2 s$ (only depending on $s$ ) such that

$$
\left|u(x)-H(x)+\frac{1}{2 s W^{\prime \prime}(0)} \frac{x}{|x|^{2 s+1}}\right| \leqslant \frac{C}{|x|^{\kappa}}, \quad \text { for }|x| \geqslant 1,
$$

and

$$
\frac{c}{|x|^{1+2 s}} \leqslant u^{\prime}(x) \leqslant \frac{C}{|x|^{1+2 s}} \quad \text { for }|x| \geqslant 1
$$


Proof. The existence of a unique solution of (1.7) is proven in [13], see also [1]. Estimate (2.1) is proven in [8] for $s=\frac{1}{2}$ and in [4], [3] respectively for $s \in\left(\frac{1}{2}, 1\right)$ and $s \in\left(0, \frac{1}{2}\right)$. Finally, estimate $(2.2)$ is shown in [1].

Next, we introduce the function $\psi$ to be the solution of

$$
\left\{\begin{array}{l}
\mathcal{I}_{s} \psi-W^{\prime \prime}(u) \psi=u^{\prime}+\eta\left(W^{\prime \prime}(u)-W^{\prime \prime}(0)\right) \text { in } \mathbb{R} \\
\psi(-\infty)=0=\psi(+\infty),
\end{array}\right.
$$

where $u$ is the solution of $(1.7)$ and

$$
\eta:=\frac{1}{W^{\prime \prime}(0)} \int_{\mathbb{R}}\left(u^{\prime}(x)\right)^{2} d x=\frac{1}{\gamma \beta} .
$$

For a detailed heuristic motivation of equation (2.3), see Section 3.1 of [8]. For later purposes, we recall the following decay estimate on the solution of (2.3):

Lemma 2.2. Assume that (1.5) holds, then there exists a unique solution $\psi$ to $(2.3)$. Furthermore $\psi \in C_{\text {loc }}^{1, \alpha}(\mathbb{R}) \cap L^{\infty}(\mathbb{R})$ for some $\alpha \in(0,1)$ and there exists $C>0$ such that for any $x \in \mathbb{R}$

$$
\left|\psi^{\prime}(x)\right| \leqslant \frac{C}{1+|x|^{1+2 s}} .
$$

Proof. The existence of a unique solution of $\left[2.3\right.$ is proven in [8] for $s=\frac{1}{2}$ and in [4], [3] respectively for $s \in\left(\frac{1}{2}, 1\right)$ and $s \in\left(0, \frac{1}{2}\right)$. Estimate 2.5) is shown in [15].

\section{Proof of Theorem 1.1}

This section is devoted to the completion of the proof of Theorem 1.1. Some arguments presented will be valid also for the case of three particles. Therefore, to make the arguments shorter, we state these auxiliary results in the course of the proof and we postpone their proof to Section 8 (in that occasion, we will then prove in a single step the results needed for both the cases of two and three particles).

The proof of Theorem 1.1 is based on the construction of auxiliary barriers for the dislocation function and in a careful use of the maximum principle. Roughly speaking, when we are close to the collision time, we can take a transition layer that goes "upwards" (respectively, "downwards") and place it a bit to the left (respectively, right) with respect to the collision point, and use them as barriers to control the original behavior of the dislocation function.

Of course, to make this argument rigorous, one has to control the small errors produced by the fact that the particle dynamics is only an approximation of the motion of the level sets of the dislocation function, and by all possible error terms that a nonlocal equation could, in principle, propagate.

Thus, to complete the proof of Theorem 1.1, as firstly seen in [8, 4, 3], we consider an auxiliary small parameter $\delta>0$ and define $\left(\bar{x}_{1}(t), \bar{x}_{2}(t)\right)$ to be the solution of the system

$$
\left\{\begin{array}{l}
\dot{\bar{x}}_{1}=\gamma\left(-\frac{\bar{x}_{1}-\bar{x}_{2}}{2 s\left|\bar{x}_{1}-\bar{x}_{2}\right|^{2 s+1}}-\sigma\left(t, \bar{x}_{1}\right)-\delta\right) \\
\dot{\bar{x}}_{2}=\gamma\left(-\frac{\bar{x}_{2}-\bar{x}_{1}}{2 s\left|\bar{x}_{2}-\bar{x}_{1}\right|^{2 s+1}}+\sigma\left(t, \bar{x}_{2}\right)+\delta\right) \\
\text { in }\left(0, T_{c}^{\delta}\right) \\
\bar{x}_{1}(0)=x_{1}^{0}-\delta, \bar{x}_{2}(0)=x_{2}^{0}+\delta
\end{array}\right.
$$


where $T_{c}^{\delta}$ is the collision time of the perturbed system (3.1), see Figure 3.

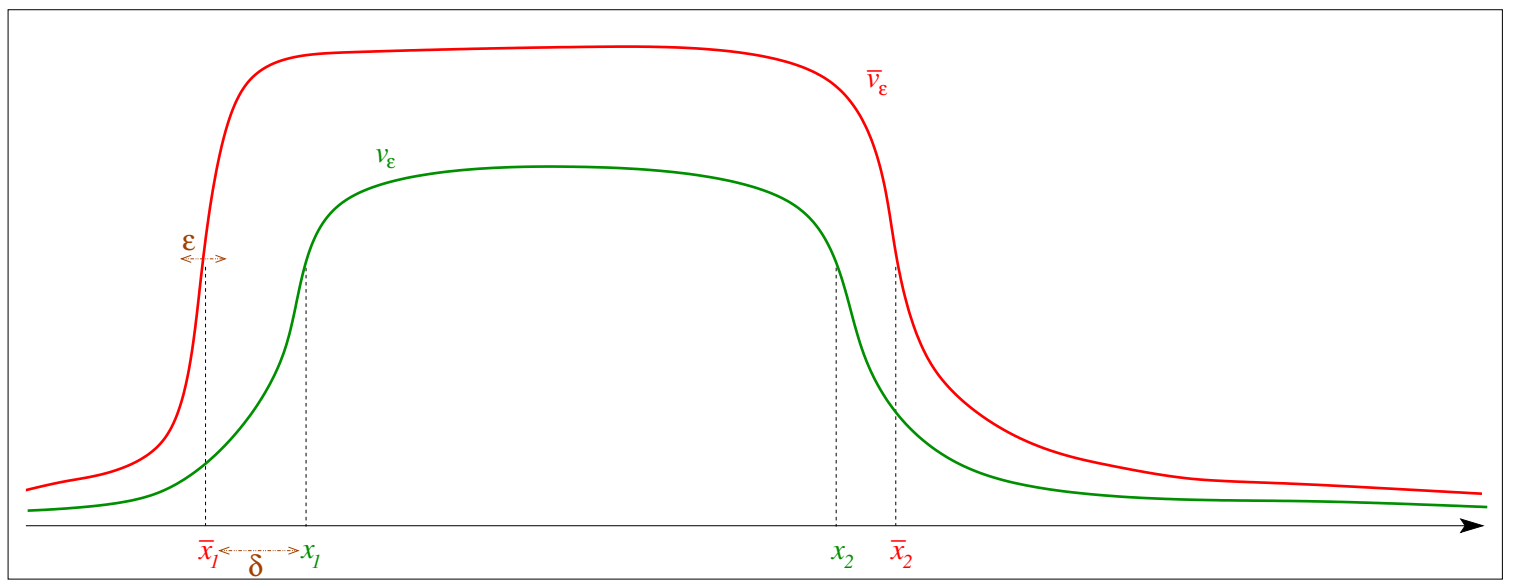

Figure 3: The geometry involved in system (3.1).

Since $\bar{x}_{1}(t)<\bar{x}_{2}(t)$ for any $t \in\left[0, T_{c}^{\delta}\right)$, system (3.1) can be rewritten in the following way

$$
\begin{cases}\dot{\bar{x}}_{1}=\gamma\left(\frac{1}{2 s\left(\bar{x}_{2}-\bar{x}_{1}\right)^{2 s}}-\sigma\left(t, \bar{x}_{1}\right)-\delta\right) & \text { in }\left(0, T_{c}^{\delta}\right) \\ \dot{\bar{x}}_{2}=\gamma\left(-\frac{1}{2 s\left(\bar{x}_{2}-\bar{x}_{1}\right)^{2 s}}+\sigma\left(t, \bar{x}_{2}\right)+\delta\right) & \text { in }\left(0, T_{c}^{\delta}\right) \\ \bar{x}_{1}(0)=x_{1}^{0}-\delta, \bar{x}_{2}(0)=x_{2}^{0}+\delta . & \end{cases}
$$

Roughly speaking, the intention of this $\delta$-perturbation is to place the particle $\bar{x}_{1}$ "slightly to the left" with respect to the original particle $x_{1}$, and the particle $\bar{x}_{2}$ "slightly to the right" with respect to the original particle $x_{2}$. This slight modification will allow to center some auxiliary transition layers in $\bar{x}_{1}$ and $\bar{x}_{2}$ and use them as barriers (as a matter of fact, this technique requires a small additional adjustment via the corrector $\psi$ introduced in (2.3), so the reader has to wait till formula $(3.10)$ for the rigorous introduction of the correct barrier). The role of the additional $\delta$-perturbation is, in a sense, to "desingularize" the problem at the collision time: that is, while the original problem experiences a collision at time $T_{c}$, the perturbed problem is still nonsingular and it can provide two-side bounds on the original dislocation function.

In order to measure the distance between the perturbed particles $\bar{x}_{1}$ and $\bar{x}_{2}$, we also denote

$$
\bar{\vartheta}(t):=\bar{x}_{2}(t)-\bar{x}_{1}(t)
$$

and

then $\bar{\vartheta}$ is solution of

$$
\vartheta_{0}:=x_{2}^{0}-x_{1}^{0}>0
$$

$$
\left\{\begin{array}{l}
\dot{\bar{\vartheta}}=\gamma\left(-\frac{1}{s \bar{\vartheta}^{2 s}}+\sigma\left(t, \bar{x}_{1}\right)+\sigma\left(t, \bar{x}_{2}\right)+2 \delta\right) \quad \text { in }\left(0, T_{c}^{\delta}\right) \\
\bar{\vartheta}(0)=\vartheta_{0}+2 \delta
\end{array}\right.
$$


Remark that

$$
\bar{\vartheta}(t)>0 \quad \text { for any } t \in\left[0, T_{c}^{\delta}\right)
$$

and

$$
\bar{\vartheta}\left(T_{c}^{\delta}\right)=0
$$

Now we show that the error due to the $\delta$-perturbation is small if so is $\delta$ :

Proposition 3.1. Let $\left(x_{1}, x_{2}\right)$ and $\left(\bar{x}_{1}, \bar{x}_{2}\right)$ be the solution respectively to system (1.10) and (3.2). Let $T_{c}<+\infty$ and $T_{c}^{\delta}$ be the collision time respectively of $(1.10)$ and (3.2). Then we have

$$
\lim _{\delta \rightarrow 0} T_{c}^{\delta}=T_{c}
$$

and for $i=1,2$

$$
\lim _{\delta \rightarrow 0} \bar{x}_{i}(t)=x_{i}(t) \quad \text { for any } t \in\left[0, T_{c}\right) .
$$

The proof of Proposition 3.1 is postponed to Section 8 .

Next result is a technical observation about the Hölder regularity of a function. Namely, to prove that a function is Hölder continuous, it is enough to check that a power of the function is Lipschitz continuous.

Lemma 3.2. Let $\beta \in(1,+\infty), \Omega$ be an open subset of $\mathbb{R}^{n}$ and $f: \Omega \rightarrow[0,+\infty)$. Let $\alpha:=1 / \beta$ and $g:=f^{\beta}$. Assume that $g$ is Lipschitz continuous in $\Omega$. Then $f \in C^{\alpha}(\Omega)$.

Proof. For any $t>0$, we set

$$
h(t):=t^{-1}\left((1+t)^{\alpha}-1\right)^{\beta} .
$$

We observe that $(1+t)^{\alpha}=1+\alpha t+O\left(t^{2}\right)$ for small $t$, therefore $h(t)=t^{-1}\left(\alpha t+O\left(t^{2}\right)\right)^{\beta}=$ $\alpha^{\beta} t^{\beta-1}(1+O(t))^{\beta}$ for small $t$ and so, since $\beta>1$,

$$
\lim _{t \rightarrow 0^{+}} h(t)=0 .
$$

Also,

Accordingly, we have that

$$
\lim _{t \rightarrow+\infty} h(t)=\lim _{t \rightarrow+\infty}\left(\left(t^{-1}+1\right)^{\alpha}-t^{-\alpha}\right)^{\beta}=1 .
$$

$$
S:=\sup _{t>0} h(t) \in[1,+\infty) .
$$

Now we show that $f \in C^{\alpha}(\Omega)$. Since $g$ is bounded, so is $f$, thus we only need to control the Hölder seminorm of $f$. For this, if the Lipschitz seminorm of $g$ is bounded by $L$, we claim that, for every $x, y \in \Omega$,

$$
|f(x)-f(y)| \leqslant(S L)^{\alpha}|x-y|^{\alpha} .
$$

To prove (3.7), we fix $x, y \in \Omega$ and we suppose, without loss of generality, that $f(x) \geqslant$ $f(y)$. In addition, if $f(y)=0$, we have that also $g(y)=0$ and then

$$
|f(x)-f(y)|=f(x)=(g(x))^{\alpha}=|g(x)-g(y)|^{\alpha} \leqslant L^{\alpha}|x-y|^{\alpha},
$$

which implies (3.7) in this case. As a consequence, we can also suppose that $f(y)>0$. Then also $g(y)>0$ and we can define

$$
t:=\frac{g(x)-g(y)}{g(y)} .
$$


By construction $t \geqslant 0$ and

$$
(1+t)^{\alpha}=\left(\frac{g(x)}{g(y)}\right)^{\alpha}=\frac{f(x)}{f(y)} .
$$

Accordingly,

$$
\begin{gathered}
|f(x)-f(y)|^{\beta}=(f(y))^{\beta}\left(\frac{f(x)}{f(y)}-1\right)^{\beta}=g(y)\left((1+t)^{\alpha}-1\right)^{\beta} \\
=g(y) t h(t)=(g(x)-g(y)) h(t) \leqslant S L|x-y|,
\end{gathered}
$$

which implies 3.7 .

Now we exploit Lemma 3.2 to obtain the Hölder continuity of the function $\bar{\vartheta}$ which was introduced in 3.3 .

Proposition 3.3. Let $\left(\bar{x}_{1}, \bar{x}_{2}\right)$ be the solution to system (3.2). Then, for any $0 \leqslant \delta \leqslant 1$ the function $\bar{\vartheta}$ defined by (3.3) is Hölder continuous in $\left[0, T_{c}^{\delta}\right]$ with Hölder constant uniform in $\delta$.

Proof. First remark that $\bar{\vartheta}$ is uniformly bounded in $\left[0, T_{c}^{\delta}\right]$. Indeed, by 3.5 there exists $T>0$ independent of $\delta$ such that $T_{c}^{\delta} \leqslant T$. Then from (3.4) we infer that for any $t \in\left[0, T_{c}^{\delta}\right]$

$$
0 \leqslant \bar{\vartheta}(t) \leqslant \vartheta_{0}+2 \delta+2 \gamma\left(\|\sigma\|_{\infty}+\delta\right) T_{c}^{\delta} \leqslant \vartheta_{0}+2+2 \gamma\left(\|\sigma\|_{\infty}+1\right) T .
$$

Next, again from $(3.4)$ we see that the function

$$
v:=(\bar{\vartheta})^{2 s+1}
$$

is solution of

$$
\dot{v}=\frac{\gamma(2 s+1)}{s}\left[-1+\left(\sigma\left(t, \bar{x}_{1}\right)+\sigma\left(t, \bar{x}_{2}\right)+2 \delta\right) s \bar{\vartheta}^{2 s}\right] \quad \text { in }\left(0, T_{c}^{\delta}\right) .
$$

Using that $\sigma$ is bounded and (3.8), we get

$$
|\dot{v}| \leqslant C
$$

where $C$ does not depend on $\delta$. Therefore $v$ is Lipschitz continuous in $\left[0, T_{c}^{\delta}\right]$ uniformly in $\delta$. The conclusion of the proposition then follows from Lemma 3.2

Next, we set

$$
\bar{c}_{i}(t):=\dot{\bar{x}}_{i}(t), \quad i=1,2
$$

and

$$
\bar{\sigma}:=\frac{\sigma+\delta}{W^{\prime \prime}(0)}
$$

Let $u$ and $\psi$ be respectively the solution of 1.7$)$ and 2.3 . We define

$$
\begin{aligned}
\bar{v}_{\varepsilon}(t, x) & :=\varepsilon^{2 s} \bar{\sigma}(t, x)+u\left(\frac{x-\bar{x}_{1}(t)}{\varepsilon}\right)+u\left(\frac{\bar{x}_{2}(t)-x}{\varepsilon}\right)-1 \\
& -\varepsilon^{2 s} \bar{c}_{1}(t) \psi\left(\frac{x-\bar{x}_{1}(t)}{\varepsilon}\right)+\varepsilon^{2 s} \bar{c}_{2}(t) \psi\left(\frac{\bar{x}_{2}(t)-x}{\varepsilon}\right) .
\end{aligned}
$$

The next two results show that, choosing conveniently $\delta=\delta_{\varepsilon}$ in $(3.2)$, the function $\bar{v}_{\varepsilon}$, defined in (3.10), is a supersolution of 1.3 provided that $\bar{x}_{1}$ and $\bar{x}_{2}$ are far enough. 
Proposition 3.4. There exist $\varepsilon_{0}>0$ and $\vartheta_{\varepsilon}, \delta_{\varepsilon}>0$ with

$$
\vartheta_{\varepsilon}, \delta_{\varepsilon}, \varepsilon \vartheta_{\varepsilon}^{-1}=o(1) \quad \text { as } \varepsilon \rightarrow 0
$$

such that for any $\varepsilon<\varepsilon_{0}$, if $\left(\bar{x}_{1}, \bar{x}_{2}\right)$ is a solution of the ODE system (3.2) with $\delta \geqslant \delta_{\varepsilon}$, then the function $\bar{v}_{\varepsilon}$ defined in 3.10 satisfies

$$
\varepsilon\left(\bar{v}_{\varepsilon}\right)_{t}-\mathcal{I}_{s} \bar{v}_{\varepsilon}+\frac{1}{\varepsilon^{2 s}} W^{\prime}\left(\bar{v}_{\varepsilon}\right)-\sigma \geqslant 0
$$

for any $x \in \mathbb{R}$ and any $t \in\left(0, T_{c}^{\delta}\right)$ such that $\bar{x}_{2}(t)-\bar{x}_{1}(t) \geqslant \vartheta_{\varepsilon}$.

Lemma 3.5. Let $v_{\varepsilon}^{0}(x)$ be defined by (1.8). Then there exists $\varepsilon_{0}>0$ such that for any $\varepsilon<\varepsilon_{0}$ and $\delta_{\varepsilon}$ given by Proposition 3.4. if $\left(\bar{x}_{1}, \bar{x}_{2}\right)$ is the solution to system (3.2) with $\delta=\delta_{\varepsilon}$, then the function $\bar{v}_{\varepsilon}$ defined in (3.10) satisfies

$$
v_{\varepsilon}^{0}(x) \leqslant \bar{v}_{\varepsilon}(0, x) \quad \text { for any } x \in \mathbb{R} .
$$

The proof of Proposition 3.4 and Lemma 3.5 is postponed to Section 8 .

Now we consider the barrier function $\bar{v}_{\varepsilon}$ defined in $(3.10)$, where $\left(\bar{x}_{1}, \bar{x}_{2}\right)$ is the solution of system (3.2) in which we fix $\delta=\delta_{\varepsilon}$, with $\delta_{\varepsilon}$ given by Proposition 3.4. For $\varepsilon$ small enough, since $T_{c}^{\delta}$ is finite by (3.5) and $\bar{\vartheta}\left(T_{c}^{\delta}\right)=0$, there exists $T_{\varepsilon}^{1}>0$ such that

$$
\bar{\vartheta}\left(T_{\varepsilon}^{1}\right)=\bar{x}_{2}\left(T_{\varepsilon}^{1}\right)-\bar{x}_{1}\left(T_{\varepsilon}^{1}\right)=\vartheta_{\varepsilon}
$$

and

$$
\bar{\vartheta}(t)=\bar{x}_{2}(t)-\bar{x}_{1}(t)>\vartheta_{\varepsilon} \quad \text { for any } t<T_{\varepsilon}^{1},
$$

where $\vartheta_{\varepsilon}$ was fixed by Proposition 3.4 .

Then by Proposition 3.4 and Lemma 3.5, we have that $\bar{v}_{\varepsilon}$ is a supersolution of (1.3)-(1.8) in $\left(0, T_{\varepsilon}^{1}\right) \times \mathbb{R}$, and the comparison principle implies

$$
v_{\varepsilon}(t, x) \leqslant \bar{v}_{\varepsilon}(t, x) \quad \text { for any }(t, x) \in\left[0, T_{\varepsilon}^{1}\right] \times \mathbb{R} .
$$

Moreover, since $\vartheta_{\varepsilon}=o(1)$ we have

$$
T_{\varepsilon}^{1}=T_{c}+o(1) \quad \text { as } \varepsilon \rightarrow 0 .
$$

Indeed, if up to subsequences, $T_{\varepsilon}^{1}$ converges as $\varepsilon \rightarrow 0$ to some $T>0$, since $T_{\varepsilon}^{1} \leqslant T_{c}^{\delta_{\varepsilon}}$ then by (3.5) we have $T \leqslant T_{c}$. Suppose by contradiction that

$$
T<T_{c}
$$

Then by Proposition 3.3 and (3.11)

$$
\left|\bar{\vartheta}\left(T_{\varepsilon}^{1}\right)-\bar{\vartheta}(T)\right|=\left|\vartheta_{\varepsilon}-\bar{\vartheta}(T)\right| \leqslant C\left|T_{\varepsilon}^{1}-T\right|^{\alpha},
$$

for some $C>0$ and $\alpha \in(0,1)$ independent of $\varepsilon$. This and $(3.6)$ imply that $\vartheta(T)=0$ which is in contradiction with (3.14). Thus (3.13) is proven.

Next, to conclude the proof of Theorem 1.1, we are going to show that starting from $T_{\varepsilon}^{1}$, after a small time $t_{\varepsilon}$, the function $v_{\varepsilon}$ satisfies

$$
v_{\varepsilon}(t, x) \leqslant \varrho_{\varepsilon}
$$

for some $\varrho_{\varepsilon}=o(1)$ as $\varepsilon \rightarrow 0$. For this scope, we denote

$$
\bar{x}_{1}^{\varepsilon}:=\bar{x}_{1}\left(T_{\varepsilon}^{1}\right), \quad \bar{x}_{2}^{\varepsilon}:=\bar{x}_{2}\left(T_{\varepsilon}^{1}\right) .
$$

Remember that from 3.11

$$
\bar{x}_{2}^{\varepsilon}-\bar{x}_{1}^{\varepsilon}=\vartheta_{\varepsilon} .
$$


We show 3.15 for $x \leqslant \bar{x}_{1}^{\varepsilon}+\frac{\vartheta_{\varepsilon}}{2}$, similarly one can prove it for $x \geqslant \bar{x}_{1}^{\varepsilon}+\frac{\vartheta_{\varepsilon}}{2}$. For this aim let us introduce the following further perturbed system, for $\hat{\delta}>\delta_{\varepsilon}$

$$
\begin{cases}\dot{\hat{x}}_{1}=\gamma\left(\frac{1}{2 s\left(\hat{x}_{2}-\hat{x}_{1}\right)^{2 s}}-\sigma\left(t, \hat{x}_{1}\right)-\hat{\delta}\right) & \text { in }\left(0, T_{c}^{\hat{\delta}}\right) \\ \dot{\hat{x}}_{2}=\gamma\left(-\frac{1}{2 s\left(\hat{x}_{2}-\hat{x}_{1}\right)^{2 s}}+\sigma\left(t, \hat{x}_{2}\right)+\hat{\delta}\right) & \text { in }\left(0, T_{c}^{\hat{\delta}}\right) \\ \hat{x}_{1}(0)=\bar{x}_{1}^{\varepsilon}-\vartheta_{\varepsilon}, \hat{x}_{2}(0)=\bar{x}_{2}^{\varepsilon}+K \vartheta_{\varepsilon}, & \end{cases}
$$

for some $K>1$ to be chosen, see Figure 4 .

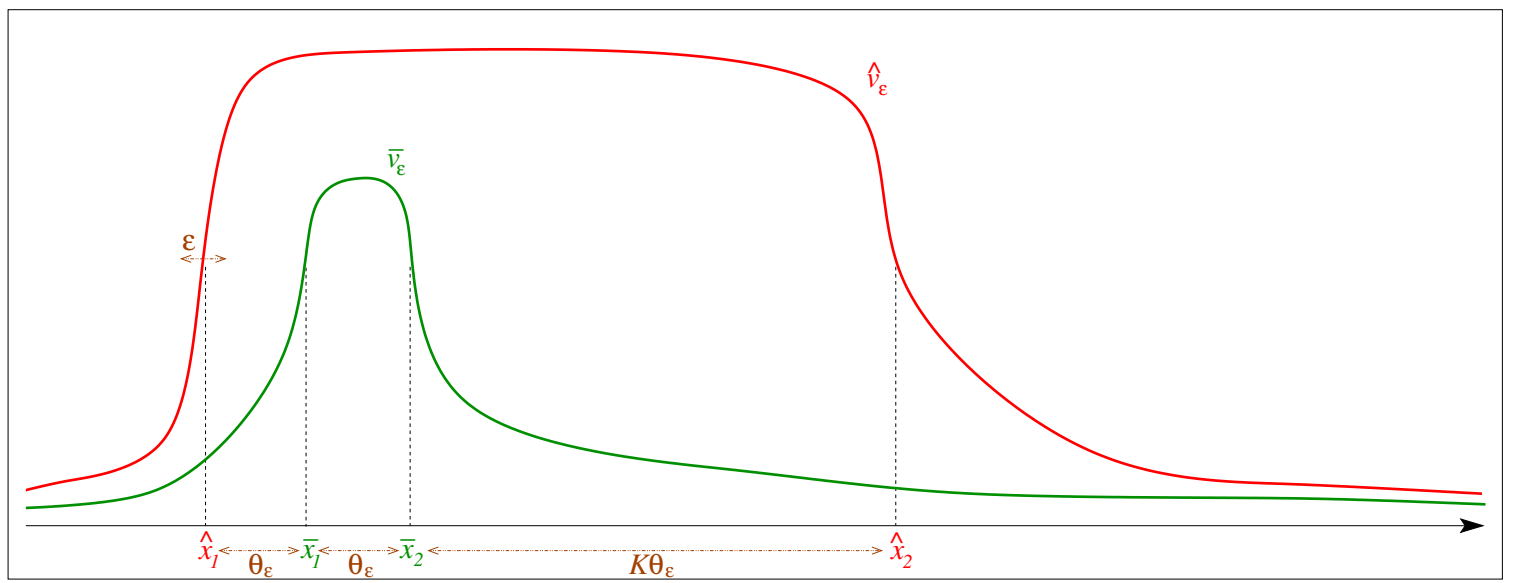

Figure 4: The geometry involved in system (3.17).

Roughly speaking, the idea behind the system in (3.17) is that at time $T_{\varepsilon}^{1}$, also the $\delta$-perturbed particles $\bar{x}_{1}$ and $\bar{x}_{2}$ that were introduced in (3.1) are close to collision. Nevertheless, these particles are "only" $\vartheta_{\varepsilon}$-close to collision, with $\vartheta_{\varepsilon}$ small, but still much larger than $\varepsilon$, thanks to Proposition 3.4. Since the excursion in the transition layers is scaled by $\varepsilon$, one can still hope to "desingularize" these $\vartheta_{\varepsilon}$-collisions. For this, it is useful to consider the "asymmetric" picture introduced in (3.17), in which the "left particle" is moved to the left by $\vartheta_{\varepsilon}$, while the "right particle" is moved to the right by a large multiple of $\vartheta_{\varepsilon}$. In this way the "middle point" between the new particles $\hat{x}_{1}$ and $\hat{x}_{2}$ introduced in (3.17) ends up to the right of the collision point of the particles $\bar{x}_{1}$ and $\bar{x}_{2}$ that were introduced in (3.1) (a formal statement will be given in Lemma 3.7). With this construction, the "tail" of the dislocation associated to the new particles $\hat{x}_{1}$ and $\hat{x}_{2}$ ends up "above" the main bump of the dislocation corresponding to the particles $\bar{x}_{1}$ and $\bar{x}_{2}$. Therefore, using the decay of the dislocation tail, the main bump of the dislocation corresponding to the particles $\bar{x}_{1}$ and $\bar{x}_{2}$ will be proved to be small.

Of course, several technicalities arise when making the above argument rigorous. For this scope, we set

$$
\hat{c}_{i}(t):=\dot{\hat{x}}_{i}(t), \quad i=1,2
$$


and

$$
\hat{\sigma}:=\frac{\sigma+\hat{\delta}}{W^{\prime \prime}(0)}
$$

We define

$$
\begin{aligned}
\hat{v}_{\varepsilon}(t, x) & :=\varepsilon^{2 s} \hat{\sigma}(t, x)+u\left(\frac{x-\hat{x}_{1}(t)}{\varepsilon}\right)+u\left(\frac{\hat{x}_{2}(t)-x}{\varepsilon}\right)-1 \\
& -\varepsilon^{2 s} \hat{c}_{1}(t) \psi\left(\frac{x-\hat{x}_{1}(t)}{\varepsilon}\right)+\varepsilon^{2 s} \hat{c}_{2}(t) \psi\left(\frac{\hat{x}_{2}(t)-x}{\varepsilon}\right),
\end{aligned}
$$

where again $u$ and $\psi$ are respectively the solution of (1.7) and (2.3). With this notation, we are in the position to estimate the modified dislocation $\bar{v}_{\varepsilon}$ at time $T_{\varepsilon}^{1}$ with the modified dislocation $\hat{v}_{\varepsilon}$ at the initial time, as stated rigorously in the next result:

Lemma 3.6. There exist $\varepsilon_{0}, \hat{\delta}_{\varepsilon}>0$ with $\delta_{\varepsilon}<\hat{\delta}_{\varepsilon}=\delta_{\varepsilon}+o(1)$ as $\varepsilon \rightarrow 0$, where $\delta_{\varepsilon}$ is given by Proposition 3.4. such that if $\left(\hat{x}_{1}, \hat{x}_{2}\right)$ is the solution to system (3.17) with $\hat{\delta}=\hat{\delta}_{\varepsilon}$, then the function $\hat{v}_{\varepsilon}$ defined in $(3.19)$ satisfies

$$
\hat{v}_{\varepsilon}(0, x) \geqslant \bar{v}_{\varepsilon}\left(T_{\varepsilon}^{1}, x\right) \quad \text { for any } x \in \mathbb{R} .
$$

The proof Lemma 3.6 is postponed to Section 8. Now we deduce some geometric consequence from Lemma 3.6, as depicted in Figure 5 and rigorously presented in the subsequent Lemma 3.7 .

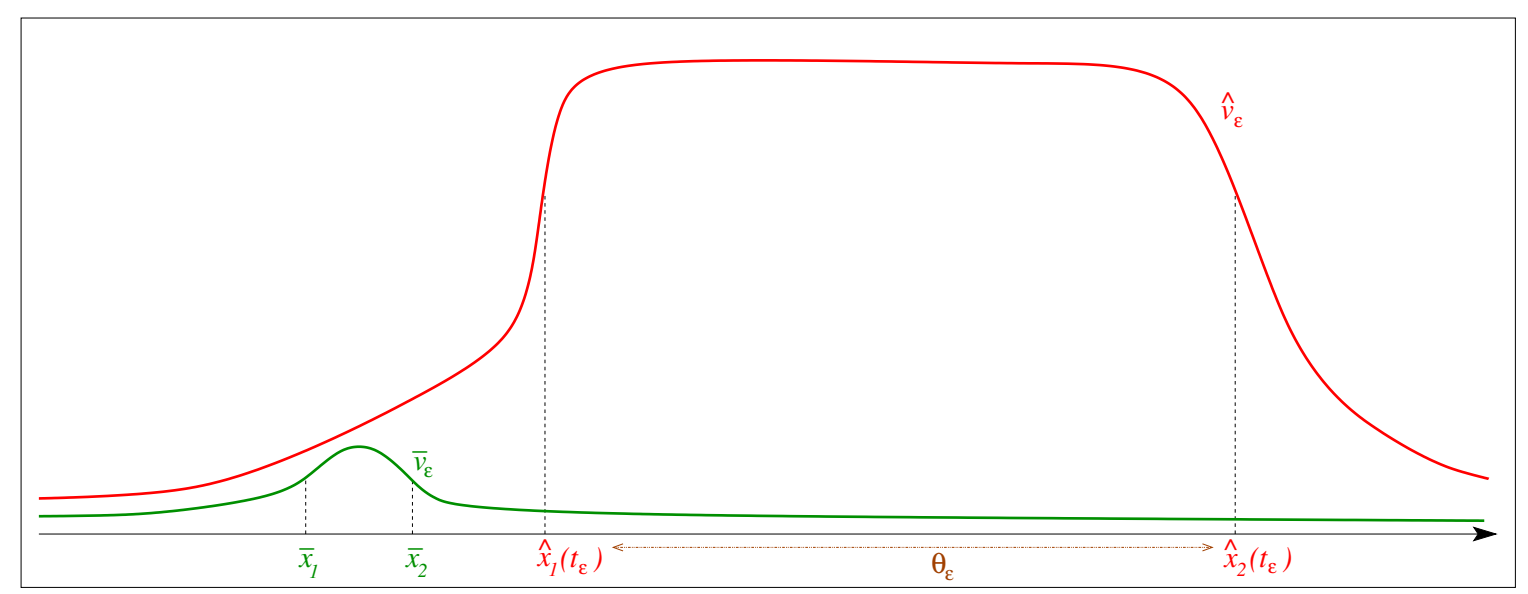

Figure 5: The geometry involved in Lemmata 3.6 and 3.7 .

Lemma 3.7. Let

$$
t_{\varepsilon}:=\frac{4 s(K+2)^{2 s} \vartheta_{\varepsilon}^{2 s+1}}{\gamma\left[1-2 s(K+2)^{2 s} \vartheta_{\varepsilon}^{2 s}\left(\|\sigma\|_{\infty}+\hat{\delta}\right)\right]} .
$$

Then there exists $K>1$ and $\varepsilon_{0}>0$ such that for any $\varepsilon<\varepsilon_{0}$ the solution $\left(\hat{x}_{1}, \hat{x}_{2}\right)$ to system (3.17) satisfies

$$
\hat{x}_{1}\left(t_{\varepsilon}\right) \geqslant \bar{x}_{2}^{\varepsilon}
$$


and for any $t \in\left[0, t_{\varepsilon}\right]$

$$
\hat{x}_{2}(t)-\hat{x}_{1}(t) \geqslant \hat{x}_{2}\left(t_{\varepsilon}\right)-\hat{x}_{1}\left(t_{\varepsilon}\right) \geqslant \vartheta_{\varepsilon}
$$

Proof. Let us denote

$$
\hat{\vartheta}(t):=\hat{x}_{2}(t)-\hat{x}_{1}(t)
$$

Then $\hat{\vartheta}(t)$ is solution of

$$
\left\{\begin{array}{l}
\dot{\hat{\vartheta}}=\gamma\left(-\frac{1}{s \hat{\vartheta}^{2 s}}+\sigma\left(t, \hat{x}_{2}\right)+\sigma\left(t, \hat{x}_{1}\right)+2 \hat{\delta}\right) \quad \text { in }\left(0, T_{c}^{\hat{\delta}}\right) \\
\hat{\vartheta}(0)=(K+2) \vartheta_{\varepsilon} .
\end{array}\right.
$$

Moreover $\hat{\vartheta}$ is a subsolution of the equation

$$
\dot{\vartheta}=\gamma\left(-\frac{1}{s \vartheta^{2 s}}+2\left(\|\sigma\|_{\infty}+\hat{\delta}\right)\right)
$$

Equation $(3.23)$ has the stationary solution $\vartheta_{s}(t)=\left[\frac{1}{2 s\left(\|\sigma\|_{\infty}+\hat{\delta}\right)}\right]^{\frac{1}{2 s}}$. Therefore for $\varepsilon$ small enough such that

$$
\hat{\vartheta}(0)=(K+2) \vartheta_{\varepsilon}<\left[\frac{1}{2 s\left(\|\sigma\|_{\infty}+\hat{\delta}\right)}\right]^{\frac{1}{2 s}},
$$

since $\hat{\vartheta}$ cannot touch $\vartheta_{s}$, its derivative remains negative. Hence for $t>0$

$$
\hat{\vartheta}(t)<(K+2) \vartheta_{\varepsilon} .
$$

Now, (3.17) and (3.24) imply

$$
\dot{\hat{x}}_{1} \geqslant\left(\frac{1}{2 s(K+2)^{2 s}\left(\vartheta_{\varepsilon}\right)^{2 s}}-\|\sigma\|_{\infty}-\hat{\delta}\right)>0 .
$$

Let $t$ be the time such that $\hat{x}_{1}(t)=\bar{x}_{2}^{\varepsilon}=\hat{x}_{1}(0)+2 \vartheta_{\varepsilon}$, then integrating $(3.25)$ in $(0, t)$ we get

from which

$$
\hat{x}_{1}(t)-\hat{x}_{1}(0)=2 \vartheta_{\varepsilon} \geqslant \gamma\left(\frac{1}{2 s(K+2)^{2 s} \vartheta_{\varepsilon}^{2 s}}-\|\sigma\|_{\infty}-\hat{\delta}\right) t
$$

$$
t \leqslant t_{\varepsilon}
$$

where $t_{\varepsilon}$ is defined in $(3.20)$.

Next, let $\tau>0$ be the time such that $\hat{\vartheta}(\tau)=\vartheta_{\varepsilon}$, then for any $t \in(0, \tau)$ we have

$$
\dot{\hat{\vartheta}} \geqslant \gamma\left(\frac{-1-2 s\|\sigma\|_{\infty} \hat{\vartheta}^{2 s}}{s \hat{\vartheta}^{2 s}}\right) \geqslant \gamma\left(\frac{-1-2 s\|\sigma\|_{\infty}(K+2)^{2 s} \vartheta_{\varepsilon}^{2 s}}{s \hat{\vartheta}^{2 s}}\right),
$$

i.e.,

$$
\hat{\vartheta}^{2 s} \dot{\hat{\vartheta}} \geqslant \frac{\gamma}{s}\left(-1-2 s\|\sigma\|_{\infty}(K+2)^{2 s} \vartheta_{\varepsilon}^{2 s}\right) .
$$

Integrating the previous inequality in $(0, \tau)$, we get $\frac{1}{2 s+1}\left(\hat{\vartheta}^{2 s+1}(\tau)-\hat{\vartheta}^{2 s+1}(0)\right)=\frac{1}{2 s+1} \vartheta_{\varepsilon}^{2 s+1}\left(1-(K+2)^{2 s+1}\right) \geqslant \frac{\gamma}{s}\left(-1-2 s\|\sigma\|_{\infty}(K+2)^{2 s} \vartheta_{\varepsilon}^{2 s}\right) \tau$, 
from which

$$
\tau \geqslant \frac{s \vartheta_{\varepsilon}^{2 s+1}\left[(K+2)^{2 s+1}-1\right]}{\gamma(2 s+1)\left(1+2 s\|\sigma\|_{\infty}(K+2)^{2 s} \vartheta_{\varepsilon}^{2 s}\right)}
$$

Comparing $\tau$ with $t_{\varepsilon}$ defined in (3.20), we see that it is possible to choose $K$ big enough so that

$$
\tau>t_{\varepsilon} .
$$

For such a choice of $K$, the monotonicity of $\hat{\vartheta}$ implies $(3.22)$. Finally $(3.21)$ is a consequence of (3.26) and the monotonicity of $\hat{x}_{1}$. This concludes the proof of the lemma.

With the auxiliary results introduced above, we are now in the position to conclude the proof of Theorem 1.1. We consider now as barrier the function $\hat{v}_{\varepsilon}$ defined in (3.19), where we fix $\hat{\delta}=\hat{\delta}_{\varepsilon}$ in system (3.17), with $\hat{\delta}_{\varepsilon}$ given by Lemma 3.6, and $K$ given by Lemma 3.7 . For $\varepsilon$ small enough, from (3.22) and Proposition 3.4, the function $\hat{v}_{\varepsilon}$ satisfies

$$
\varepsilon\left(\hat{v}_{\varepsilon}\right)_{t}-\mathcal{I}_{s} \hat{v}_{\varepsilon}+\frac{1}{\varepsilon^{2 s}} W^{\prime}\left(\hat{v}_{\varepsilon}\right)-\sigma(t, x) \geqslant 0 \quad \text { in }\left(0, t_{\varepsilon}\right) \times \mathbb{R} .
$$

Moreover from (3.12) and Lemma 3.6

$$
v_{\varepsilon}\left(T_{\varepsilon}^{1}, x\right) \leqslant \hat{v}_{\varepsilon}(0, x) \text { for any } x \in \mathbb{R} .
$$

The comparison principle then implies

$$
v_{\varepsilon}\left(T_{\varepsilon}^{1}+t, x\right) \leqslant \hat{v}_{\varepsilon}(t, x) \text { for any }(t, x) \in\left[0, t_{\varepsilon}\right] \times \mathbb{R} .
$$

Now, for $x \leqslant \bar{x}_{1}^{\varepsilon}+\frac{\vartheta_{\varepsilon}}{2}$, from 3.16), 3.21) and 3.22) we know that

$$
x-\hat{x}_{1}\left(t_{\varepsilon}\right) \leqslant-\frac{\vartheta_{\varepsilon}}{2} \quad \text { and } \quad \hat{x}_{2}\left(t_{\varepsilon}\right)-x \geqslant \frac{3 \vartheta_{\varepsilon}}{2} .
$$

Therefore, from estimate (2.1) we have

$$
u\left(\frac{x-\hat{x}_{1}\left(t_{\varepsilon}\right)}{\varepsilon}\right)+u\left(\frac{\hat{x}_{2}\left(t_{\varepsilon}\right)-x}{\varepsilon}\right)-1 \leqslant C \varepsilon^{2 s} \vartheta_{\varepsilon}^{-2 s} .
$$

Moreover (3.22), (3.17) and (3.18) imply that

$$
\left|\hat{c}_{i}\left(t_{\varepsilon}\right)\right| \leqslant C \vartheta_{\varepsilon}^{-2 s} \text {. }
$$

Finally, from (3.19), (3.27), (3.28) and (3.29), we conclude that

$$
v_{\varepsilon}\left(T_{\varepsilon}^{1}+t_{\varepsilon}, x\right) \leqslant C \varepsilon^{2 s} \vartheta_{\varepsilon}^{-2 s} \text { for any } x \leqslant \bar{x}_{1}^{\varepsilon}+\frac{\vartheta_{\varepsilon}}{2} \text {. }
$$

The same inequality for $x \geqslant \bar{x}_{1}^{\varepsilon}+\frac{\vartheta_{\varepsilon}}{2}$ can be proven considering the system (3.17) with initial condition

for $K$ large enough.

$$
\hat{x}_{1}(0)=\bar{x}_{1}^{\varepsilon}-K \vartheta_{\varepsilon}, \quad \hat{x}_{2}(0)=\bar{x}_{2}^{\varepsilon}+\vartheta_{\varepsilon}
$$

We have proven 1.12 with

$$
T_{\varepsilon}:=T_{\varepsilon}^{1}+t_{\varepsilon},
$$

$t_{\varepsilon}$ given by Lemma 3.7 with $\hat{\delta}=\hat{\delta}_{\varepsilon}$ given by Lemma 3.6 , and

$$
\varrho_{\varepsilon}=C \varepsilon^{2 s} \vartheta_{\varepsilon}^{-2 s}=o(1) \quad \text { as } \varepsilon \rightarrow 0,
$$

with $\vartheta_{\varepsilon}$ given by Proposition 3.4. Moreover from (3.13) and 3.20 we see that

$$
T_{\varepsilon}=T_{c}+o(1) \quad \text { as } \varepsilon \rightarrow 0,
$$


and this concludes the proof of Theorem 1.1 .

\section{Proof of Theorem 1.2}

We consider the function $h(\tau, \xi)$ which is solution of

$$
\left\{\begin{array}{l}
h_{\tau}+W^{\prime}(h)=0, \quad \forall \tau \in(0,+\infty) \\
h(0, \xi)=\xi
\end{array}\right.
$$

From assumptions (1.5), we have that there exists $\varepsilon_{0}>0$ such that for any $\varepsilon<\varepsilon_{0}$,

$$
W^{\prime \prime}(\xi) \geqslant \frac{W^{\prime \prime}(0)}{2}=\frac{\beta}{2}>0 \quad \text { for any }|\xi| \leqslant \varrho_{\varepsilon}
$$

and

$$
W^{\prime}(\xi)>0 \text { for any } \xi \in\left(0, \varrho_{\varepsilon}\right], \quad W^{\prime}(\xi)<0 \text { for any } \xi \in\left[-\varrho_{\varepsilon}, 0\right), \quad W^{\prime}(0)=0 .
$$

Therefore, the solution $h$ of (4.1) satisfies: $h(\tau, 0) \equiv 0 ; h(\tau, \xi)$ is positive and decreasing in $\tau$, if $\xi \in\left(0, \varrho_{\varepsilon}\right] ; h(\tau, \xi)$ is negative and increasing in $\tau$, if $\xi \in\left[-\varrho_{\varepsilon}, 0\right)$. Hence if $\xi \in\left(0, \varrho_{\varepsilon}\right]$

$$
h_{\tau}=-W^{\prime}(h) \leqslant-\frac{\beta}{2} h,
$$

which implies

$$
0<h(\tau, \xi) \leqslant \xi e^{-\frac{\beta}{2} \tau}
$$

Similarly for $\xi \in\left[-\varrho_{\varepsilon}, 0\right)$

$$
\xi e^{-\frac{\beta}{2} \tau} \leqslant h(\tau, \xi)<0
$$

Now, the function $\tilde{h}(t, x):=h\left(\frac{t-T^{\varepsilon}}{\varepsilon^{2 s+1}}, \varrho_{\varepsilon}\right)$, where $T^{\varepsilon}$ is given by Theorem 1.1, is solution of the equation (1.3) for $t>T^{\varepsilon}$, with $\tilde{h}\left(T^{\varepsilon}, x\right)=\varrho_{\varepsilon}$. Then, the comparison principle and estimate 1.12 imply

$$
v_{\varepsilon}(t, x) \leqslant \tilde{h}(t, x) \text { for any } x \in \mathbb{R}, t>T^{\varepsilon},
$$

and from 4.2 we get

$$
v_{\varepsilon}(t, x) \leqslant \varrho_{\varepsilon} e^{-\frac{\beta}{2} \frac{t-T^{\varepsilon}}{\varepsilon^{2} s+1}} \quad \text { for any } x \in \mathbb{R}, t>T^{\varepsilon} .
$$

Finally, using (2.1), it is easy to check that the initial datum (1.8) satisfies

$$
v_{\varepsilon}^{0}(x) \geqslant-C \varepsilon^{2 s} \text { for any } x \in \mathbb{R},
$$

therefore, we can similarly prove that

$$
v_{\varepsilon}(t, x) \geqslant-\varrho_{\varepsilon} e^{-\frac{\beta}{2} \frac{t-T^{\varepsilon}}{\varepsilon^{2 s+1}}} \quad \text { for any } x \in \mathbb{R}, t>T^{\varepsilon},
$$

and this concludes the proof of Theorem 1.2 , 


\section{Proof of Theorem 1.3}

We consider an auxiliary small parameter $\delta>0$ and define $\left(\bar{x}_{1}(t), \bar{x}_{2}(t), \bar{x}_{3}(t)\right)$ to be the solution to system: for $i=1,2,3$

$$
\left\{\begin{array}{l}
\dot{\bar{x}}_{i}=\gamma\left(\sum_{j \neq i} \zeta_{i} \zeta_{j} \frac{\bar{x}_{i}-\bar{x}_{j}}{2 s\left|\bar{x}_{i}-\bar{x}_{j}\right|^{1+2 s}}-\zeta_{i} \sigma\left(t, \bar{x}_{i}\right)-\zeta_{i} \delta\right) \quad \text { in }\left(0, T_{c}^{\delta}\right) \\
\bar{x}_{i}(0)=x_{i}^{0}-\zeta_{i} \delta,
\end{array}\right.
$$

where $T_{c}^{\delta}$ is the collision time of the perturbed system (5.1), see Figure 6 .

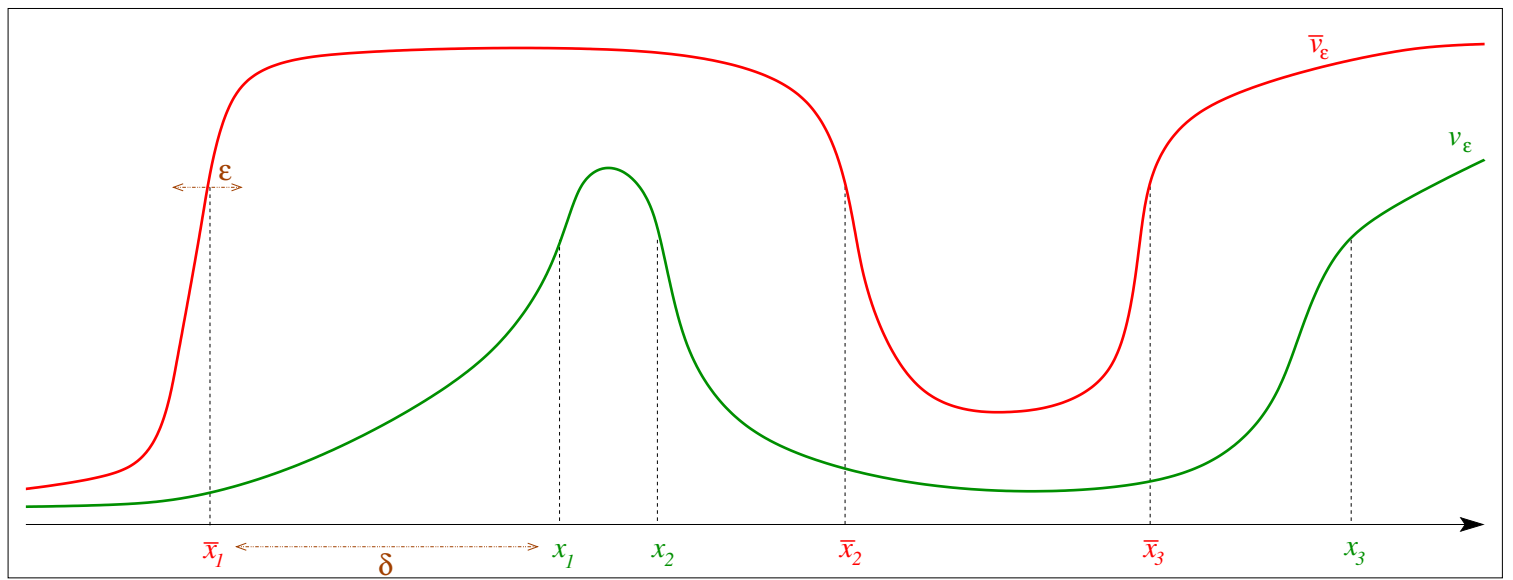

Figure 6: The geometry involved in system (5.1).

In a sense, the system in (5.1) is the analogue, in the case of three particles, of the system that was introduced in (3.1). As in that case, the scope of (5.1) is to "remove the singularity" caused by the collision in the original system. Of course, in the case of three particles, an additional difficulty arises, since the new particles need to be moved either to the left or to the right, depending on the orientations of the original dislocations. In particular, in the case of three particles, in order to obtain bounds both by above and by below, one also needs another system with the opposite sign convention (this additional system will be introduced in formula (5.13) below).

Let us denote for $i=1,2$

$$
\bar{\vartheta}_{i}(t):=\bar{x}_{i+1}(t)-\bar{x}_{i}(t)
$$

and

$$
\vartheta_{0}^{i}:=x_{i+1}^{0}-x_{i}^{0}>0
$$


then $\left(\bar{\vartheta}_{1}, \bar{\vartheta}_{2}\right)$ is solution of

$$
\left\{\begin{array}{l}
\dot{\bar{\vartheta}}_{1}=\frac{\gamma}{s}\left(-\frac{1}{\left.\bar{\vartheta}_{1}^{2 s}+\frac{1}{2\left(\bar{\vartheta}_{1}+\bar{\vartheta}_{2}\right)^{2 s}}+\frac{1}{2 \bar{\vartheta}_{2}^{2 s}}+s \sigma\left(t, x_{1}\right)+s \sigma\left(t, x_{2}\right)+2 s \delta\right)} \quad \text { in }\left(0, T_{c}^{\delta}\right)\right. \\
\dot{\bar{\vartheta}}_{2}=\frac{\gamma}{s}\left(\frac{1}{2 \vartheta_{1}^{2 s}}+\frac{1}{2\left(\bar{\vartheta}_{1}+\bar{\vartheta}_{2}\right)^{2 s}}-\frac{1}{\bar{\vartheta}_{2}^{2 s}}-s \sigma\left(t, x_{3}\right)-s \sigma\left(t, x_{2}\right)-2 s \delta\right) \\
\bar{\vartheta}_{1}(0)=\vartheta_{1}^{0}+2 \delta>0 \\
\bar{\vartheta}_{2}(0)=\vartheta_{2}^{0}-2 \delta>0 .
\end{array}\right.
$$

In the next result we will show that the error introduced by the $\delta$-perturbation remains small in the trajectory and does not affect too much the collision time. The precise statement goes as follows.

Proposition 5.1. Let $\left(x_{1}, x_{2}, x_{3}\right)$ and $\left(\bar{x}_{1}, \bar{x}_{2}, \bar{x}_{3}\right)$ be the solution respectively of system (1.15) and (5.1). Let $T_{c}<+\infty$ and $T_{c}^{\delta}$ be the collision time respectively of (1.15) and (5.1). Then we have

$$
\lim _{\delta \rightarrow 0} T_{c}^{\delta}=T_{c}
$$

and for $i=1,2,3$

$$
\lim _{\delta \rightarrow 0} \bar{x}_{i}(t)=x_{i}(t) \quad \text { for any } t \in\left[0, T_{c}\right) .
$$

The proof of Proposition 5.1 is postponed to Section 8 (notice that Proposition 5.1 is the generalization of Proposition 3.1 to the case of three particles: we kept the two statement separate for the sake of clarity, but the proof will consider both the cases at the same time).

Now we show that the minimum between $\bar{\vartheta}_{1}$ and $\bar{\vartheta}_{2}$ is Hölder continuous:

Proposition 5.2. Let $\left(\bar{x}_{1}, \bar{x}_{2}, \bar{x}_{3}\right)$ be the solution to system (5.1) and $\left(\bar{\vartheta}_{1}, \bar{\vartheta}_{2}\right)$ given by (5.2). Then, for any $0 \leqslant \delta \leqslant 1$ the function $\min \left\{\bar{\vartheta}_{1}, \bar{\vartheta}_{2}\right\}$ is Hölder continuous in $\left[0, T_{c}^{\delta}\right]$ with Hölder constant uniform in $\delta$.

Proof. First remark that $\bar{\vartheta}_{1}$ and $\bar{\vartheta}_{2}$ are uniformly bounded in $\left[0, T_{c}^{\delta}\right]$. Indeed, by (5.4) there exists $T>0$ independent of $\delta$ such that $T_{c}^{\delta} \leqslant T$. Moreover, by (5.3)

$$
\begin{aligned}
\dot{\bar{\vartheta}}_{1}+\dot{\bar{\vartheta}}_{2} & =\frac{\gamma}{s}\left(-\frac{1}{2 \bar{\vartheta}_{1}^{2 s}}+\frac{1}{2\left(\bar{\vartheta}_{1}+\bar{\vartheta}_{2}\right)^{2 s}}-\frac{1}{2 \bar{\vartheta}_{2}^{2 s}}+\frac{1}{2\left(\bar{\vartheta}_{1}+\bar{\vartheta}_{2}\right)^{2 s}}+s \sigma\left(t, x_{1}\right)-s \sigma\left(t, x_{3}\right)\right) \\
& \leqslant 2 \gamma\|\sigma\|_{\infty} .
\end{aligned}
$$

Therefore

$$
0 \leqslant \bar{\vartheta}_{1}+\bar{\vartheta}_{2} \leqslant \vartheta_{1}^{0}+\vartheta_{2}^{0}+2 \gamma\|\sigma\|_{\infty} T_{c}^{\delta} \leqslant \vartheta_{1}^{0}+\vartheta_{2}^{0}+2 \gamma\|\sigma\|_{\infty} T
$$

Next, let us denote

$$
\bar{\vartheta}_{m}(t):=\min _{i=1,2} \bar{\vartheta}_{i}(t)=\bar{\vartheta}_{i(t)}(t) .
$$

Then from (5.3) we infer that

$$
\begin{aligned}
\bar{\vartheta}_{m}(t+h)-\bar{\vartheta}_{m}(t) & =\bar{\vartheta}_{i(t+h)}(t+h)-\bar{\vartheta}_{i(t)}(t) \leqslant \bar{\vartheta}_{i(t)}(t+h)-\bar{\vartheta}_{i(t)}(t) \\
& \leqslant C \int_{t}^{t+h}\left(\frac{1}{\bar{\vartheta}_{m}^{2 s}(\tau)}+1\right) d \tau
\end{aligned}
$$


and

$$
\begin{aligned}
\bar{\vartheta}_{m}(t+h)-\bar{\vartheta}_{m}(t) & =\bar{\vartheta}_{i(t+h)}(t+h)-\bar{\vartheta}_{i(t)}(t) \geqslant \bar{\vartheta}_{i(t+h)}(t+h)-\bar{\vartheta}_{i(t+h)}(t) \\
& \geqslant-C \int_{t}^{t+h}\left(\frac{1}{\bar{\vartheta}_{m}^{2 s}(\tau)}+1\right) d \tau .
\end{aligned}
$$

Now, let us denote

$$
v:=\left(\bar{\vartheta}_{m}\right)^{2 s+1}
$$

Then we have

$$
\begin{aligned}
\left|\frac{v(t+h)-v(t)}{h}\right| & =(2 s+1)\left(\xi_{h} \bar{\vartheta}_{m}(t+h)+\left(1-\xi_{h}\right) \bar{\vartheta}_{m}(t)\right)^{2 s}\left|\frac{\bar{\vartheta}_{m}(t+h)-\bar{\vartheta}_{m}(t)}{h}\right| \\
& \leqslant(2 s+1)\left(\xi_{h} \bar{\vartheta}_{m}(t+h)+\left(1-\xi_{h}\right) \bar{\vartheta}_{m}(t)\right)^{2 s} \frac{C}{h} \int_{t}^{t+h}\left(\frac{1}{\bar{\vartheta}_{m}^{2 s}(\tau)}+1\right) d \tau,
\end{aligned}
$$

for some $\xi_{h} \in[0,1]$. Passing to the limit as $h \rightarrow 0$, we get

$$
\limsup _{h \rightarrow 0}\left|\frac{v(t+h)-v(t)}{h}\right| \leqslant C,
$$

i.e. the function $v$ is Lipschitz continuous in $\left[0, T_{c}^{\delta}\right]$ uniformly in $\delta$. The conclusion of the proposition then follows from Lemma 3.2 .

Next, we set

$$
\bar{c}_{i}(t):=\dot{\bar{x}}_{i}(t), \quad i=1,2,3
$$

and

$$
\bar{\sigma}:=\frac{\sigma+\delta}{W^{\prime \prime}(0)}
$$

Let $u$ and $\psi$ be respectively the solution of $(1.7)$ and $(2.3)$. We define

$$
\bar{v}_{\varepsilon}(t, x):=\varepsilon^{2 s} \bar{\sigma}(t, x)+\sum_{i=1}^{3} u\left(\zeta_{i} \frac{x-\bar{x}_{i}(t)}{\varepsilon}\right)-1-\sum_{i=1}^{3} \zeta_{i} \varepsilon^{2 s} \bar{c}_{i}(t) \psi\left(\zeta_{i} \frac{x-\bar{x}_{i}(t)}{\varepsilon}\right) .
$$

Under the appropriate choice of the parameters, the function $\bar{v}_{\varepsilon}$ is a supersolution of (1.3)-(1.14), as next results point out:

Proposition 5.3. There exist $\varepsilon_{0}>0$ and $\vartheta_{\varepsilon}, \delta_{\varepsilon}>0$ with

$$
\vartheta_{\varepsilon}, \delta_{\varepsilon}, \varepsilon \vartheta_{\varepsilon}^{-1}=o(1) \quad \text { as } \varepsilon \rightarrow 0
$$

such that for any $\varepsilon<\varepsilon_{0}$, if $\left(\bar{x}_{1}, \bar{x}_{2}, \bar{x}_{3}\right)$ is a solution of the ODE system in (5.1) with $\delta \geqslant \delta_{\varepsilon}$, then the function $\bar{v}_{\varepsilon}$ defined in (5.8) satisfies

$$
\varepsilon\left(\bar{v}_{\varepsilon}\right)_{t}-\mathcal{I}_{s} \bar{v}_{\varepsilon}+\frac{1}{\varepsilon^{2 s}} W^{\prime}\left(\bar{v}_{\varepsilon}\right)-\sigma \geqslant 0
$$

for any $x \in \mathbb{R}$ and any $t \in\left(0, T_{c}^{\delta}\right)$ such that $\bar{x}_{i+1}(t)-\bar{x}_{i}(t) \geqslant \vartheta_{\varepsilon}$ for $i=1,2$. 
Lemma 5.4. Let $v_{\varepsilon}^{0}(x)$ be defined by (1.14). Then there exists $\varepsilon_{0}>0$ such that for any $\varepsilon<\varepsilon_{0}$ and $\delta_{\varepsilon}$ given by Proposition 5.3, if $\left(\bar{x}_{1}, \bar{x}_{2}, \bar{x}_{3}\right)$ is the solution to system (5.1) with $\delta=\delta_{\varepsilon}$, then the function $\bar{v}_{\varepsilon}$ defined in (5.8) satisfies

$$
v_{\varepsilon}^{0}(x) \leqslant \bar{v}_{\varepsilon}(0, x) \quad \text { for any } x \in \mathbb{R} .
$$

The proof of Proposition 5.3 and Lemma 5.4 is postponed to Section 8 . We observe that Proposition 5.3 and Lemma 5.4 are the generalization, respectively, of Proposition 3.4 and Lemma 3.5 to the case of three particles (the proof presented in Section 8 will indeed work simultaneously for the cases of two and three particles).

Now we consider the barrier function $\bar{v}_{\varepsilon}$ defined in (5.8), where $\left(\bar{x}_{1}, \bar{x}_{2}, \bar{x}_{3}\right)$ is the solution to system (5.1) in which we fix $\delta=\delta_{\varepsilon}$, with $\delta_{\varepsilon}$ given by Proposition 5.3. For $\varepsilon$ small enough, since $T_{c}^{\delta}$ is finite by (5.4), there exists $\bar{T}_{\varepsilon}^{1}>0$ such that

$$
\min _{i=1,2} \bar{x}_{i+1}\left(\bar{T}_{\varepsilon}^{1}\right)-\bar{x}_{i}\left(\bar{T}_{\varepsilon}^{1}\right)=\vartheta_{\varepsilon}
$$

and

$$
\bar{x}_{i+1}(t)-\bar{x}_{i}(t)>\vartheta_{\varepsilon} \quad \text { for any } t<\bar{T}_{\varepsilon}^{1}, \quad i=1,2 .
$$

Without loss of generality, we may assume

$$
\min _{i=1,2} \bar{x}_{i+1}\left(\bar{T}_{\varepsilon}^{1}\right)-\bar{x}_{i}\left(\bar{T}_{\varepsilon}^{1}\right)=\bar{x}_{2}\left(\bar{T}_{\varepsilon}^{1}\right)-\bar{x}_{1}\left(\bar{T}_{\varepsilon}^{1}\right)=\vartheta_{\varepsilon} .
$$

From (5.1), 5.6) and (5.9), we infer that

$$
\left|\bar{c}_{i}\left(\bar{T}_{\varepsilon}^{1}\right)\right| \leqslant C \vartheta_{\varepsilon}^{-2 s} .
$$

By Proposition 5.3 and Lemma 5.4, the function $\bar{v}_{\varepsilon}$ defined in (5.8), is a supersolution of (1.3)-(1.14) in $\left(0, \bar{T}_{\varepsilon}^{1}\right) \times \mathbb{R}$, and the comparison principle implies

$$
v_{\varepsilon}(t, x) \leqslant \bar{v}_{\varepsilon}(t, x) \quad \text { for any }(t, x) \in\left[0, \bar{T}_{\varepsilon}^{1}\right] \times \mathbb{R} .
$$

Moreover, since $\vartheta_{\varepsilon}=o(1)$ as $\varepsilon \rightarrow 0$, as in Section 3, from Propositions 5.1 and 5.2, we have

$$
\bar{T}_{\varepsilon}^{1}=T_{c}+o(1) \quad \text { as } \varepsilon \rightarrow 0 .
$$

Similarly, for $\delta>0$, one can define $\left(\underline{x}_{1}, \underline{x}_{2}, \underline{x}_{3}\right)$ to be the solution of the system

$$
\left\{\begin{array}{l}
\underline{\dot{x}}_{i}=\gamma\left(\sum_{j \neq i} \zeta_{i} \zeta_{j} \frac{\underline{x}_{i}-\underline{x}_{j}}{2 s\left|\underline{x}_{i}-\underline{x}_{j}\right|^{1+2 s}}-\zeta_{i} \sigma\left(t, \underline{x}_{i}\right)+\zeta_{i} \delta\right) \\
\underline{x}_{i}(0)=x_{i}^{0}+\zeta_{i} \delta
\end{array}\right.
$$

see Figure 7. 


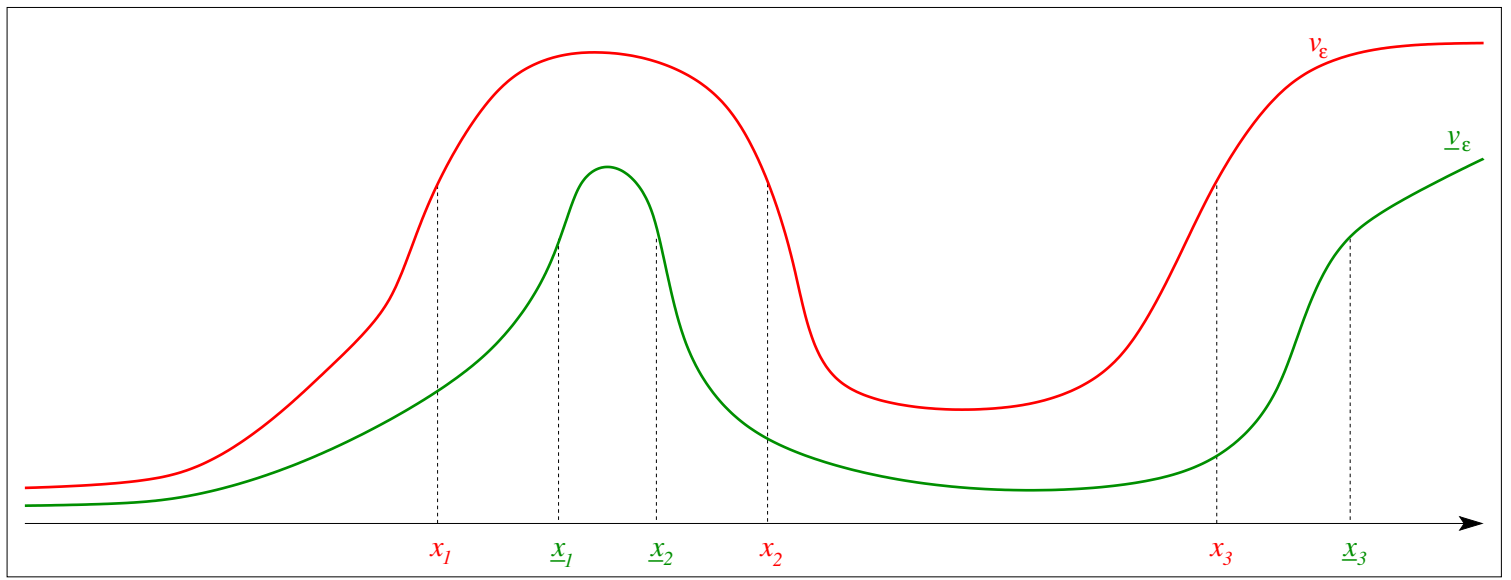

Figure 7: The geometry involved in system (5.13).

Let also

$$
\underline{v}_{\varepsilon}(t, x):=\varepsilon^{2 s} \frac{\sigma(t, x)-\delta}{W^{\prime \prime}(0)}+\sum_{i=1}^{3} u\left(\zeta_{i} \frac{x-\underline{x}_{i}(t)}{\varepsilon}\right)-1-\sum_{i=1}^{3} \zeta_{i} \varepsilon^{2 s} \underline{x}_{i}(t) \psi\left(\zeta_{i} \frac{x-\underline{x}_{i}(t)}{\varepsilon}\right) .
$$

Then, one can prove that there exists $\delta_{\varepsilon}=o(1)$ as $\varepsilon \rightarrow 0$ and $\underline{T}_{\varepsilon}^{2}$ such that

$$
\begin{gathered}
\underline{T}_{\varepsilon}^{2}=T_{c}+o(1) \quad \text { as } \varepsilon \rightarrow 0, \\
\min _{i=1,2} \underline{x}_{i+1}\left(\underline{T}_{\varepsilon}^{2}\right)-\underline{x}_{i}\left(\underline{T}_{\varepsilon}^{2}\right)=\underline{x}_{2}\left(\underline{T}_{\varepsilon}^{2}\right)-\underline{x}_{1}\left(\underline{T}_{\varepsilon}^{2}\right)=\vartheta_{\varepsilon}, \\
\left|\underline{x}_{i}\left(\underline{T}_{\varepsilon}^{2}\right)\right| \leqslant C \vartheta_{\varepsilon}^{-2 s}
\end{gathered}
$$

and

$$
v_{\varepsilon}(t, x) \geqslant \underline{v}_{\varepsilon}(t, x) \quad \text { for any }(t, x) \in\left[0, \underline{T}_{\varepsilon}^{2}\right] \times \mathbb{R} .
$$

In what follows, we will denote

$$
\bar{x}_{i}^{\varepsilon}:=\bar{x}_{i}\left(\bar{T}_{\varepsilon}^{1}\right),
$$

and

$$
\underline{x}_{i}^{\varepsilon}:=\underline{x}_{i}\left(\underline{T}_{\varepsilon}^{2}\right) .
$$

Roughly speaking, in this case, the dislocation function will be the superposition of three transition layers: the idea is now to deal separately with the annihilation of two of them, by possibly moving the transition point if necessary (this adjustment of the transition point uses the quantities $\bar{x}_{i}^{\varepsilon}$ and $\underline{x}_{i}^{\varepsilon}$ that we have just introduced). The formal statement goes as follows:

Lemma 5.5. For any $x \in \mathbb{R}$ we have

$$
\sum_{i=2}^{3} u\left(\zeta_{i} \frac{x-\bar{x}_{i}^{\varepsilon}}{\varepsilon}\right)-1 \leqslant C \varepsilon^{2 s} \vartheta_{\varepsilon}^{-2 s}
$$


and

$$
\sum_{i=1}^{2} u\left(\zeta_{i} \frac{x-\underline{x}_{i}^{\varepsilon}}{\varepsilon}\right)-1 \geqslant-C \varepsilon^{2 s} \vartheta_{\varepsilon}^{-2 s} .
$$

Proof. Let us prove (5.17). Let us first suppose that there exists $k=2,3$ such that

$$
\left|x-\bar{x}_{k}^{\varepsilon}\right| \leqslant \frac{\vartheta_{\varepsilon}}{2} \text {. }
$$

Then, from $(5.9)$ for $j \neq k$

$$
\zeta_{j}\left(x-\bar{x}_{j}^{\varepsilon}\right) \leqslant-\frac{\vartheta_{\varepsilon}}{2}
$$

and estimate 2.1) implies

$$
u\left(\zeta_{j} \frac{x-\bar{x}_{j}^{\varepsilon}}{\varepsilon}\right) \leqslant C \varepsilon^{2 s} \vartheta_{\varepsilon}^{-2 s} .
$$

Therefore, we have

$$
\sum_{i=2}^{3} u\left(\zeta_{i} \frac{x-\bar{x}_{i}^{\varepsilon}}{\varepsilon}\right)-1 \leqslant u\left(\zeta_{j} \frac{x-\bar{x}_{j}^{\varepsilon}}{\varepsilon}\right) \leqslant C \varepsilon^{2 s} \vartheta_{\varepsilon}^{-2 s} .
$$

Next, if for any $i=2,3$

$$
\left|x-\bar{x}_{k}^{\varepsilon}\right| \geqslant \frac{\vartheta_{\varepsilon}}{2}
$$

then again estimate (2.1) implies (5.17). Similarly one can prove (5.18).

From (5.16), (5.18) and (5.15), we infer that

$$
v_{\varepsilon}\left(\underline{T}_{\varepsilon}^{2}, x\right) \geqslant u\left(\frac{x-\underline{x}_{3}^{\varepsilon}}{\varepsilon}\right)-C \varepsilon^{2 s} \vartheta_{\varepsilon}^{-2 s} \text { for any } x \in \mathbb{R},
$$

which proves (1.17) with

$$
T_{\varepsilon}^{2}=\underline{T}_{\varepsilon}^{2}, \quad z_{\varepsilon}=\underline{x}_{3}^{\varepsilon}, \quad \text { and } \quad \varrho_{\varepsilon}=C \varepsilon^{2 s} \vartheta_{\varepsilon}^{-2 s} .
$$

Now, to prove (1.16), let us divide the proof in two cases, depending on whether we are in a simple or in triple collision.

5.1. Case 1: simple collision. In this case (up to renaming the particles), the first two particles gets to collision while the third one remains far enough. More precisely, let us suppose that

$$
\bar{x}_{2}^{\varepsilon}-\bar{x}_{1}^{\varepsilon}=\vartheta_{\varepsilon}, \quad \bar{x}_{3}^{\varepsilon}-\bar{x}_{2}^{\varepsilon} \geqslant M \vartheta_{\varepsilon},
$$

with $M>2$ independent of $\varepsilon$ to be determined. Let us introduce the following further perturbed system, for $\hat{\delta}>\delta_{\varepsilon}$ and $1<K<M-1$ :

$$
\left\{\begin{array}{l}
\dot{\hat{x}}_{i}=\gamma\left(\sum_{j \neq i} \zeta_{i} \zeta_{j} \frac{\hat{x}_{i}-\hat{x}_{j}}{2 s\left|\hat{x}_{i}-\hat{x}_{j}\right|^{1+2 s}}-\zeta_{i} \sigma\left(t, \hat{x}_{i}\right)-\zeta_{i} \hat{\delta}\right) \quad \text { in }\left(0, T_{c}^{\hat{\delta}}\right) \\
\hat{x}_{1}(0)=\bar{x}_{1}^{\varepsilon}-\vartheta_{\varepsilon}, \hat{x}_{2}(0)=\bar{x}_{2}^{\varepsilon}+K \vartheta_{\varepsilon}, \hat{x}_{3}(0)=\bar{x}_{3}^{\varepsilon}-\vartheta_{\varepsilon},
\end{array}\right.
$$

where $T_{c}^{\hat{\delta}}$ is the collision time of the system (5.21).

Roughly speaking, the idea behind the system in (5.21) is that, for simple collisions, one can adapt the technique introduced in (3.17) for the case of two collisions. That is, 
we can move the first particle slightly to the left and the second particle slightly to the right. As done in (3.17), the right displacement of the second particle, though small, is a large multiple of the left displacement of the first particle (this is needed to construct barriers from above). Since, in this case, the third particle is far from the collision, this construction leaves "space enough" to move the third particle slightly to the left, without producing new collisions in this procedure.

Of course, the technical details in this case are more complicated than in the case of two particles and the notation becomes somehow heavier, since it must comprise not only one additional particles, but also the different orientations of the dislocations involved. So, to make the argument rigorous, we set

$$
\hat{c}_{i}(t):=\dot{\hat{x}}_{i}(t), \quad i=1,2,3
$$

and

$$
\hat{\sigma}:=\frac{\sigma+\hat{\delta}}{W^{\prime \prime}(0)}
$$

We define

$$
\hat{v}_{\varepsilon}(t, x):=\varepsilon^{2 s} \hat{\sigma}(t, x)+\sum_{i=1}^{3} u\left(\zeta_{i} \frac{x-\hat{x}_{i}(t)}{\varepsilon}\right)-1-\sum_{i=1}^{3} \zeta_{i} \varepsilon^{2 s} \hat{c}_{i}(t) \psi\left(\zeta_{i} \frac{x-\hat{x}_{i}(t)}{\varepsilon}\right),
$$

where again $u$ and $\psi$ are respectively the solution of $(1.7)$ and $(2.3)$.

Lemma 5.6. There exist $\varepsilon_{0}, \hat{\delta}_{\varepsilon}>0$ with $\delta_{\varepsilon}<\hat{\delta}_{\varepsilon}=\delta_{\varepsilon}+o(1)$ as $\varepsilon \rightarrow 0$, where $\delta_{\varepsilon}$ is given by Proposition 5.3, such that if $\left(\hat{x}_{1}, \hat{x}_{2}, \hat{x}_{3}\right)$ is the solution to system (5.21) with $\hat{\delta}=\hat{\delta}_{\varepsilon}$, then the function $\hat{v}_{\varepsilon}$ defined in (5.24) satisfies

$$
\hat{v}_{\varepsilon}(0, x) \geqslant \bar{v}_{\varepsilon}\left(\bar{T}_{\varepsilon}^{1}, x\right) \quad \text { for any } x \in \mathbb{R} .
$$

The proof of Lemma 5.6 is postponed to Section 8. Using Lemma 5.6, we obtain the geometric consequences depicted in Figure 8 and formally described in the forthcoming Lemma 5.7.

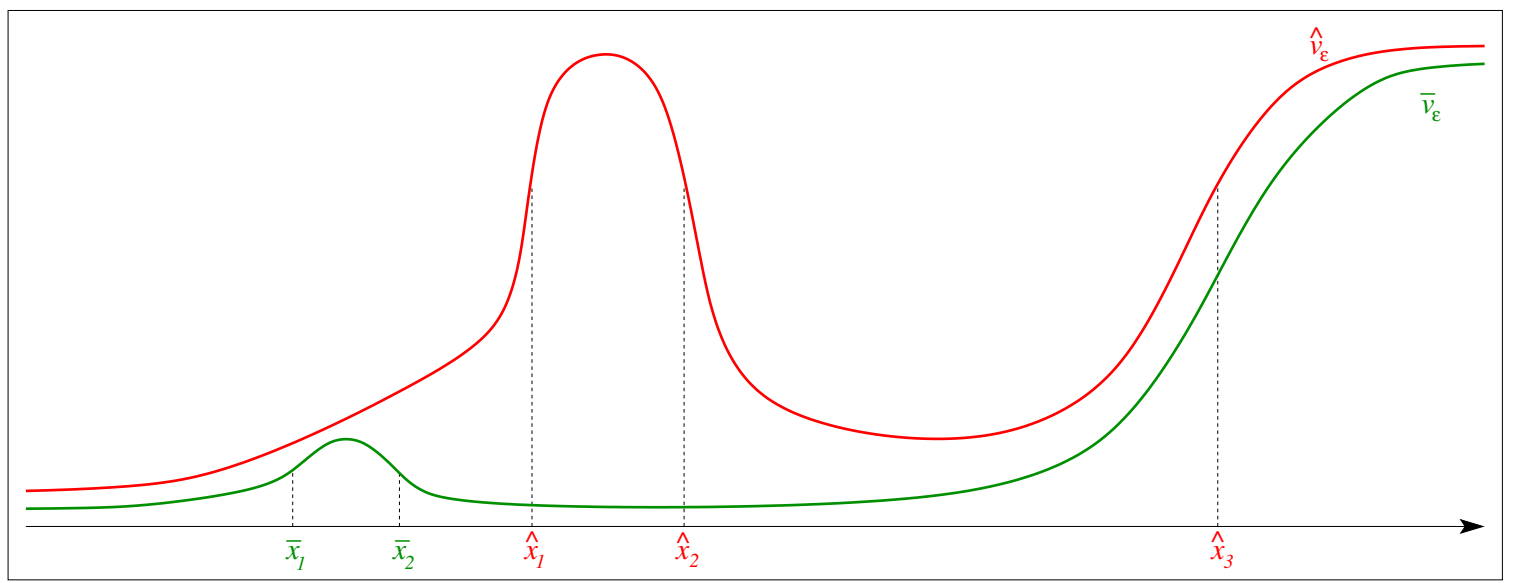

Figure 8: The geometry involved in Lemmata 5.6 and 5.7. 
Lemma 5.7. Let

$$
t_{\varepsilon}:=\frac{2^{2 s+2} s(K+2)^{2 s} \vartheta_{\varepsilon}^{2 s+1}}{\gamma\left[2^{2 s}-1-2 s^{2 s+1}(K+2)^{2 s} \vartheta_{\varepsilon}^{2 s}\left(\|\sigma\|_{\infty}+\hat{\delta}\right)\right]} .
$$

Then there exist $K, M>1$ and $\varepsilon_{0}>0$ such that for any $\varepsilon<\varepsilon_{0}$ the solution $\left(\hat{x}_{1}, \hat{x}_{2}, \hat{x}_{3}\right)$ to system (5.21) satisfies

$$
\hat{x}_{2}(t)-\hat{x}_{1}(t) \text { is decreasing for any } t>0,
$$

$$
\hat{x}_{1}\left(t_{\varepsilon}\right) \geqslant \bar{x}_{2}^{\varepsilon},
$$

and for any $t \in\left[0, t_{\varepsilon}\right]$

$$
\hat{x}_{3}(t)-\hat{x}_{2}(t) \geqslant \hat{x}_{2}(t)-\hat{x}_{1}(t) \geqslant \vartheta_{\varepsilon} .
$$

The proof of Lemma 5.7 is rather long and technical, therefore, not to interrupt the flow of ideas at this point, before giving the proof of Lemma 5.7, let us conclude the proof of Theorem 1.3 (the proof of Lemma 5.7 will then presented in detail in Subsection 5.3).

So, let $K$ and $M$ be given by Lemma 5.7. Let us suppose that the second inequality in (5.20) is satisfied with such a $M$. We consider as barrier the function $\hat{v}_{\varepsilon}$ defined in (5.24), where we fix $\hat{\delta}=\hat{\delta}_{\varepsilon}$ in system (5.21), with $\hat{\delta}_{\varepsilon}$ given by Lemma 5.6. From (5.28) and Proposition 5.3 we infer that the function $\hat{v}_{\varepsilon}$ satisfies

$$
\varepsilon\left(\hat{v}_{\varepsilon}\right)_{t}-\mathcal{I}_{s} \hat{v}_{\varepsilon}+\frac{1}{\varepsilon^{2 s}} W^{\prime}\left(\hat{v}_{\varepsilon}\right)-\sigma(t, x) \geqslant 0 \quad \text { in }\left(0, t_{\varepsilon}\right) \times \mathbb{R} .
$$

Moreover from (5.11) and Lemma 5.6

$$
v_{\varepsilon}\left(\bar{T}_{\varepsilon}^{1}, x\right) \leqslant \hat{v}_{\varepsilon}(0, x) \text { for any } x \in \mathbb{R} .
$$

The comparison principle then implies

$$
v_{\varepsilon}\left(\bar{T}_{\varepsilon}^{1}+t, x\right) \leqslant \hat{v}_{\varepsilon}(t, x) \text { for any }(t, x) \in\left[0, t_{\varepsilon}\right] \times \mathbb{R} .
$$

Now, for $x \leqslant \bar{x}_{1}^{\varepsilon}+\frac{\vartheta_{\varepsilon}}{2}$, from (5.20), (5.27) and (5.28) we know that

$$
x-\hat{x}_{1}\left(t_{\varepsilon}\right) \leqslant-\frac{\vartheta_{\varepsilon}}{2}, \text { and } \hat{x}_{2}\left(t_{\varepsilon}\right)-x \geqslant \frac{3 \vartheta_{\varepsilon}}{2} .
$$

Therefore, from estimate (2.1) we have

$$
\sum_{i=1}^{2} u\left(\zeta_{i} \frac{x-\hat{x}_{i}\left(t_{\varepsilon}\right)}{\varepsilon}\right)-1 \leqslant C \varepsilon^{2 s} \vartheta_{\varepsilon}^{-2 s} .
$$

Moreover, from (5.28), (5.21) and (5.22), we infer that for $i=1,2,3$

$$
\left|\hat{c}_{i}\left(t_{\varepsilon}\right)\right| \leqslant C \vartheta_{\varepsilon}^{-2 s} \text {. }
$$

Finally, from (5.24), (5.29), (5.30) and (5.31), we conclude that

$$
v_{\varepsilon}\left(\bar{T}_{\varepsilon}^{1}+t_{\varepsilon}, x\right) \leqslant C \varepsilon^{2 s} \vartheta_{\varepsilon}^{-2 s}+u\left(\frac{x-\hat{x}_{3}\left(t_{\varepsilon}\right)}{\varepsilon}\right) \quad \text { for any } x \leqslant \bar{x}_{1}^{\varepsilon}+\frac{\vartheta_{\varepsilon}}{2} .
$$

A similar inequality for $x \geqslant \bar{x}_{1}^{\varepsilon}+\frac{\vartheta_{\varepsilon}}{2}$ can be proven considering the solution $\left(\hat{\hat{x}}_{1}, \hat{\hat{x}}_{2}, \hat{\hat{x}}_{3}\right)$ to system (5.21) with initial condition

$$
\hat{\hat{x}}_{1}(0)=\bar{x}_{1}^{\varepsilon}-K \vartheta_{\varepsilon}, \quad \hat{\hat{x}}_{2}(0)=\bar{x}_{2}^{\varepsilon}+\vartheta_{\varepsilon}, \quad \hat{\hat{x}}_{3}(0)=\bar{x}_{3}^{\varepsilon}-\vartheta_{\varepsilon} .
$$


Therefore, for $y_{\varepsilon}=\min \left\{\hat{x}_{3}\left(t_{\varepsilon}\right), \hat{\hat{x}}_{3}\left(t_{\varepsilon}\right)\right\}$ we have

$$
v_{\varepsilon}\left(\bar{T}_{\varepsilon}^{1}+t_{\varepsilon}, x\right) \leqslant C \varepsilon^{2 s} \vartheta_{\varepsilon}^{-2 s}+u\left(\frac{x-y_{\varepsilon}}{\varepsilon}\right) \quad \text { for any } x \in \mathbb{R} .
$$

This proves 1.16) with

$$
T_{\varepsilon}^{1}=\bar{T}_{\varepsilon}^{1}+t_{\varepsilon}, \quad y_{\varepsilon}=\min \left\{\hat{x}_{3}\left(t_{\varepsilon}\right), \hat{\hat{x}}_{3}\left(t_{\varepsilon}\right)\right\} \quad \text { and } \quad \varrho_{\varepsilon}=C \varepsilon^{2 s} \vartheta_{\varepsilon}^{-2 s} .
$$

Recalling (5.19), from (5.5) we infer that $\left|y_{\varepsilon}-z_{\varepsilon}\right|=o(1)$ as $\varepsilon \rightarrow 0$. This concludes the proof of Theorem 1.3 in Case 1.

5.2. Case 2: close to a triple collision. In this case, let us suppose that

$$
\bar{x}_{2}^{\varepsilon}-\bar{x}_{1}^{\varepsilon}=\vartheta_{\varepsilon}, \quad \bar{x}_{3}^{\varepsilon}-\bar{x}_{2}^{\varepsilon} \leqslant M \vartheta_{\varepsilon},
$$

where $M$ is given by Lemma 5.7. From (5.8), (5.11), (5.10) and (5.17) we infer that for any $x \in \mathbb{R}$

i.e. 1.16 with

$$
v^{\varepsilon}\left(\bar{T}_{\varepsilon}^{1}, x\right) \leqslant u\left(\frac{x-\bar{x}_{1}^{\varepsilon}}{\varepsilon}\right)+C \varepsilon^{2 s} \vartheta_{\varepsilon}^{-2 s}
$$

$$
y_{\varepsilon}=\bar{x}_{1}^{\varepsilon}, \quad T_{\varepsilon}=\bar{T}_{\varepsilon}^{1} \quad \text { and } \quad \varrho_{\varepsilon}=C \varepsilon^{2 s} \vartheta_{\varepsilon}^{-2 s} .
$$

Remark that from $(5.32)$ and $(5.5)$ we have

$$
\left|z_{\varepsilon}-y_{\varepsilon}\right|=\left|\underline{x}_{3}^{\varepsilon}-\bar{x}_{1}^{\varepsilon}\right| \leqslant\left|\underline{x}_{3}^{\varepsilon}-\bar{x}_{3}^{\varepsilon}\right|+\left|\bar{x}_{3}^{\varepsilon}-\bar{x}_{1}^{\varepsilon}\right| \leqslant\left|\underline{x}_{3}^{\varepsilon}-\bar{x}_{3}^{\varepsilon}\right|+(M+1) \vartheta_{\varepsilon}=o(1) \quad \text { as } \varepsilon \rightarrow 0 \text {. }
$$

This concludes the proof of Theorem 1.3 in Case 2 .

It only remains to prove Lemma 5.7. We will do this in the subsequent subsection.

5.3. Proof of Lemma 5.7. Let us denote for $i=1,2$

$$
\hat{\vartheta}_{i}(t):=\hat{x}_{i+1}(t)-\hat{x}_{i}(t)
$$

where $\left(\hat{x}_{1}(t), \hat{x}_{2}(t), \hat{x}_{3}(t)\right)$ is the solution to system (5.21). Then, recalling (5.20), we see that $\left(\hat{\vartheta}_{1}, \hat{\vartheta}_{2}\right)$ satisfies

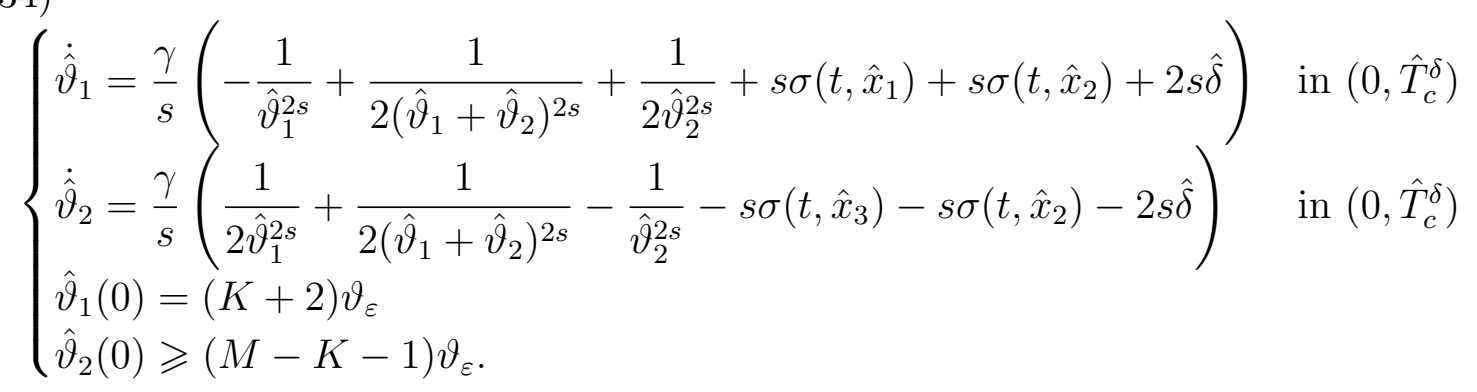

Lemma 5.8. For any $K>1$ there exists $M>2 K+3$ independent of $\varepsilon$ and $\varepsilon_{0}>0$ such that for any $\varepsilon<\varepsilon_{0},\left(\hat{\vartheta}_{1}, \hat{\vartheta}_{2}\right)$ defined in (5.33) satisfies

$$
\hat{\vartheta}_{1}(t) \leqslant \hat{\vartheta}_{2}(t), \quad \text { for any } t>0,
$$

and

$$
\hat{\vartheta}_{1}(t) \text { is decreasing for any } t>0 \text {. }
$$


Proof. For $K>1$ and $M>2 K+3$, let us denote

$$
a(M):=\frac{K+2}{M-K-1} \geqslant \frac{\hat{\vartheta}_{1}(0)}{\hat{\vartheta}_{2}(0)} .
$$

For fixed $K$, let us choose $M$ so big and $\varepsilon$ so small such that

$$
-1+a(M)^{2 s}+a(M)^{2 s+1}+2 s\left(\|\sigma\|_{\infty}+\hat{\delta}\right)(a(M)+1)(K+2)^{2 s} \vartheta_{\varepsilon}^{2 s}<0 .
$$

We want to show that for any $t>0$

$$
\frac{\hat{\vartheta}_{1}(t)}{\hat{\vartheta}_{2}(t)} \leqslant a(M) \leqslant 1
$$

From system (5.34), we infer that $\hat{\vartheta}_{1}$ satisfies

$$
\dot{\hat{\vartheta}}_{1} \leqslant \frac{\gamma}{s}\left(-\frac{1}{\hat{\vartheta}_{1}^{2 s}}+\frac{1}{\hat{\vartheta}_{2}^{2 s}}+2 s\|\sigma\|_{\infty}+2 s \hat{\delta}\right)=\frac{\gamma}{s \hat{\vartheta}_{1}^{2 s}}\left[-1+\frac{\hat{\vartheta}_{1}^{2 s}}{\hat{\vartheta}_{2}^{2 s}}+2 s\left(\|\sigma\|_{\infty}+\hat{\delta}\right) \hat{\vartheta}_{1}^{2 s}\right]
$$

and

$$
\dot{\hat{\vartheta}}_{2} \geqslant \frac{\gamma}{s}\left(-\frac{1}{\hat{\vartheta}_{2}^{2 s}}-2 s\|\sigma\|_{\infty}-2 s \hat{\delta}\right) .
$$

From (5.37) we see that the right-hand side in $(5.39)$ is negative at $t=0$. We deduce that there exists $T>0$, that we choose maximal, such that

$$
\dot{\hat{\vartheta}}_{1}(t) \leqslant 0 \quad \text { for any } t \in(0, T) \text {. }
$$

Then

$$
\hat{\vartheta}_{1}(t) \leqslant(K+2) \vartheta_{\varepsilon} \quad \text { for any } t \in(0, T)
$$

moreover in $(0, T)$ we have that

$$
\begin{aligned}
\frac{d}{d t}\left(\frac{\hat{\vartheta}_{1}}{\hat{\vartheta}_{2}}\right) & =\frac{\dot{\hat{\vartheta}}_{1} \hat{\vartheta}_{2}-\hat{\vartheta}_{1} \dot{\hat{\vartheta}}_{2}}{\hat{\vartheta}_{2}^{2}} \\
& \leqslant \frac{\gamma}{s \hat{\vartheta}_{2}^{2}}\left[-\frac{\hat{\vartheta}_{2}}{\hat{\vartheta}_{1}^{2 s}}+\frac{\hat{\vartheta}_{2}}{\hat{\vartheta}_{2}^{2 s}}+\frac{\hat{\vartheta}_{1}}{\hat{\vartheta}_{2}^{2 s}}+2 s\left(\|\sigma\|_{\infty}+\hat{\delta}\right)\left(\hat{\vartheta}_{1}+\hat{\vartheta}_{2}\right)\right] \\
& =\frac{\gamma \hat{\vartheta}_{2}}{s \hat{\vartheta}_{2}^{2} \hat{\vartheta}_{1}^{2 s}}\left[-1+\frac{\hat{\vartheta}_{1}^{2 s}}{\hat{\vartheta}_{2}^{2 s}}+\frac{\hat{\vartheta}_{1}^{2 s+1}}{\hat{\vartheta}_{2}^{2 s+1}}+2 s\left(\|\sigma\|_{\infty}+\hat{\delta}\right)\left(\hat{\vartheta}_{1}+\hat{\vartheta}_{2}\right) \frac{\hat{\vartheta}_{1}^{2 s}}{\hat{\vartheta}_{2}}\right] \\
& =\frac{\gamma \hat{\vartheta}_{2}}{s \hat{\vartheta}_{2}^{2} \hat{\vartheta}_{1}^{2 s}}\left[-1+\frac{\hat{\vartheta}_{1}^{2 s}}{\hat{\vartheta}_{2}^{2 s}}+\frac{\hat{\vartheta}_{1}^{2 s+1}}{\hat{\vartheta}_{2}^{2 s+1}}+2 s\left(\|\sigma\|_{\infty}+\hat{\delta}\right)\left(\frac{\hat{\vartheta}_{1}}{\hat{\vartheta}_{2}}+1\right) \hat{\vartheta}_{1}^{2 s}\right] \\
& \leqslant \frac{\gamma \hat{\vartheta}_{2}}{s \hat{\vartheta}_{2}^{2} \hat{\vartheta}_{1}^{2 s}}\left[-1+\frac{\hat{\vartheta}_{1}^{2 s}}{\hat{\vartheta}_{2}^{2 s}}+\frac{\hat{\vartheta}_{1}^{2 s+1}}{\hat{\vartheta}_{2}^{2 s+1}}+2 s\left(\|\sigma\|_{\infty}+\hat{\delta}\right)\left(\frac{\hat{\vartheta}_{1}}{\hat{\vartheta}_{2}}+1\right)(K+2)^{2 s} \vartheta_{\varepsilon}^{2 s}\right]
\end{aligned}
$$


Integrating in $(0, t)$, we infer that for any $t \in(0, T)$

$$
\begin{aligned}
\frac{\hat{\vartheta}_{1}(t)}{\hat{\vartheta}_{2}(t)} \leqslant a(M)+\int_{0}^{t} \frac{\gamma \hat{\vartheta}_{2}(\tau)}{s \hat{\vartheta}_{2}^{2}(\tau) \hat{\vartheta}_{1}^{2 s}(\tau)}\left[-1+\frac{\hat{\vartheta}_{1}^{2 s}(\tau)}{\hat{\vartheta}_{2}^{2 s}(\tau)}+\frac{\hat{\vartheta}_{1}^{2 s+1}(\tau)}{\hat{\vartheta}_{2}^{2 s+1}(\tau)}\right. \\
\left.+2 s\left(\|\sigma\|_{\infty}+\hat{\delta}\right)\left(\frac{\hat{\vartheta}_{1}(\tau)}{\hat{\vartheta}_{2}(\tau)}+1\right)(K+2)^{2 s} \vartheta_{\varepsilon}^{2 s}\right] d \tau .
\end{aligned}
$$

Let us call

$$
g(\tau):=-1+\frac{\hat{\vartheta}_{1}^{2 s}(\tau)}{\hat{\vartheta}_{2}^{2 s}(\tau)}+\frac{\hat{\vartheta}_{1}^{2 s+1}(\tau)}{\hat{\vartheta}_{2}^{2 s+1}(\tau)}+2 s\left(\|\sigma\|_{\infty}+\hat{\delta}\right)\left(\frac{\hat{\vartheta}_{1}(\tau)}{\hat{\vartheta}_{2}(\tau)}+1\right)(K+2)^{2 s} \vartheta_{\varepsilon}^{2 s} .
$$

We observe that $g(0)<0$ thanks to (5.37). Thus, we want to show that

$$
g(\tau)<0 \quad \text { for any } \tau \in(0, T) .
$$

Assume by contradiction that this is not true. Then there exists $t_{0} \in(0, T)$ such that

$$
g(\tau)<0 \quad \text { for any } \tau \in\left(0, t_{0}\right)
$$

and $g\left(t_{0}\right)=0$. Then $\frac{\hat{\vartheta}_{1}\left(t_{0}\right)}{\hat{\vartheta}_{2}\left(t_{0}\right)}=\bar{a}$ with

$$
-1+\bar{a}^{2 s}+\bar{a}^{2 s+1}+2 s\left(\|\sigma\|_{\infty}+\hat{\delta}\right)(\bar{a}+1)(K+2)^{2 s} \vartheta_{\varepsilon}^{2 s}=0 .
$$

On the other hand, by $(5.39)$ and $(5.43)$, we see that

$$
\dot{\hat{\vartheta}}_{1}<\frac{\gamma}{s \hat{\vartheta}_{1}^{2 s}} g<0 \quad \text { in }\left(0, t_{0}\right)
$$

and therefore, recalling $(5.40)$, we conclude that $t_{0}<T$. In particular, we can use (5.41) with $t=t_{0}$. Thus, from (5.41) and (5.43) we infer that

$$
\bar{a}=\frac{\hat{\vartheta}_{1}\left(t_{0}\right)}{\hat{\vartheta}_{2}\left(t_{0}\right)} \leqslant a(M)+\int_{0}^{t} \frac{\gamma \hat{\vartheta}_{2}(\tau)}{s \hat{\vartheta}_{2}^{2}(\tau) \hat{\vartheta}_{1}^{2 s}(\tau)} g(\tau) d \tau<a(M) .
$$

This and (5.44) give

$$
\begin{aligned}
0 & =-1+\bar{a}^{2 s}+\bar{a}^{2 s+1}+2 s\left(\|\sigma\|_{\infty}+\hat{\delta}\right)(\bar{a}+1)(K+2)^{2 s} \vartheta_{\varepsilon}^{2 s} \\
& <-1+a(M)^{2 s}+a(M)^{2 s+1}+2 s\left(\|\sigma\|_{\infty}+\hat{\delta}\right)(a(M)+1)(K+2)^{2 s} \vartheta_{\varepsilon}^{2 s}
\end{aligned}
$$

and this is in contradiction with (5.37). Therefore we have completed the proof of $(5.42)$. In turn, we see that (5.41) and (5.42) imply (5.38), and thus (5.35). Finally, (5.36) is a consequence of $(5.40)$.

Let us now complete the proof of Lemma 5.7 .

Let us fix $K>1$ such that

$$
\frac{(K+2)^{2 s+1}-1}{(K+2)^{2 s}} \geqslant \frac{(2 s+1) 2^{2 s+2}\left(1+2^{2 s}\right)}{2^{2 s}-1} .
$$

Let us choose $M>2 K+3$ such that (5.35) and (5.36) hold for any $\varepsilon$ small enough. Then (5.26) is given by (5.36) and consequently for $t>0$

$$
\hat{\vartheta}_{1}(t)<(K+2) \vartheta_{\varepsilon},
$$


and there exists $\tau>0$ such that

$$
\hat{\vartheta}_{1}(\tau)=\vartheta_{\varepsilon}
$$

Now, from system (5.34) we see that $\hat{\vartheta}_{1}$ satisfies

$$
\dot{\hat{\vartheta}}_{1} \geqslant \frac{\gamma}{s}\left(-\frac{1}{\hat{\vartheta}_{1}^{2 s}}-2 s\|\sigma\|_{\infty}\right)=-\gamma \frac{1+2 s\|\sigma\|_{\infty} \hat{\vartheta}_{1}^{2 s}}{s \hat{\vartheta}_{1}^{2 s}} .
$$

Multiplying by $\hat{\vartheta}_{1}^{2 s}$, integrating in $(0, \tau)$ and using (5.46), we get

$$
\begin{aligned}
\frac{1}{2 s+1}\left(\hat{\vartheta}_{1}^{2 s+1}(\tau)-\hat{\vartheta}_{1}^{2 s+1}(0)\right) & =\frac{\vartheta_{\varepsilon}^{2 s+1}}{2 s+1}\left(1-(K+2)^{2 s+1}\right) \\
& \geqslant-\gamma \frac{1+2 s\|\sigma\|_{\infty}(K+2)^{2 s} \vartheta_{\varepsilon}^{2 s}}{s} \tau
\end{aligned}
$$

from which

$$
\tau \geqslant \frac{s \vartheta_{\varepsilon}^{2 s+1}\left((K+2)^{2 s+1}-1\right)}{\gamma(2 s+1)\left(1+2 s(K+2)^{2 s} \vartheta_{\varepsilon}^{2 s}\|\sigma\|_{\infty}\right)} .
$$

Next, for fixed $K$ satisfying (5.45), let $\varepsilon$ be so small that

$$
2^{2 s+1} s(K+2)^{2 s} \vartheta_{\varepsilon}^{2 s}\left(\|\sigma\|_{\infty}+\hat{\delta}\right) \leqslant \frac{2^{2 s}-1}{2} .
$$

Then, from (5.21), (5.35), 5.46) and (5.49), we have

$$
\begin{aligned}
\dot{\hat{x}}_{1} & =\gamma\left(\frac{1}{2 s \hat{\vartheta}_{1}^{2 s}}-\frac{1}{2 s\left(\hat{\vartheta}_{1}+\hat{\vartheta}_{2}\right)^{2 s}}-\sigma\left(t, \hat{x}_{1}\right)-\hat{\delta}\right) \\
& \geqslant \gamma\left(\frac{2^{2 s}-1}{2^{2 s+1} s \hat{\vartheta}_{1}^{2 s}}-\|\sigma\|_{\infty}-\hat{\delta}\right) \\
& =\gamma \frac{2^{2 s}-1-2^{2 s+1} s \hat{\vartheta}_{1}^{2 s}\left(\|\sigma\|_{\infty}+\hat{\delta}\right)}{2^{2 s+1} s \hat{\vartheta}_{1}^{2 s}} \\
& \geqslant \gamma \frac{2^{2 s}-1-2^{2 s+1} s(K+2)^{2 s} \vartheta_{\varepsilon}^{2 s}\left(\|\sigma\|_{\infty}+\hat{\delta}\right)}{2^{2 s+1} s(K+2)^{2 s} \vartheta_{\varepsilon}^{2 s}} \\
& \geqslant \gamma \frac{2^{2 s}-1}{2^{2 s+2} s(K+2)^{2 s} \vartheta_{\varepsilon}^{2 s}} \\
& >0 .
\end{aligned}
$$

Let $t>0$ be such that

$$
\hat{x}_{1}(t)=\bar{x}_{2}^{\varepsilon}=\hat{x}_{1}(0)+2 \vartheta_{\varepsilon},
$$

then integrating (5.50) in $(0, t)$, we get

$$
2 \vartheta_{\varepsilon} \geqslant \gamma \frac{2^{2 s}-1-2^{2 s+1} s(K+2)^{2 s} \vartheta_{\varepsilon}^{2 s}\left(\|\sigma\|_{\infty}+\hat{\delta}\right)}{2^{2 s+1} s(K+2)^{2 s} \vartheta_{\varepsilon}^{2 s}} t
$$

from which

$$
t \leqslant t_{\varepsilon}
$$


where $t_{\varepsilon}$ is defined in (5.25). Moreover from (5.45) and (5.49)

$$
\begin{aligned}
\frac{(K+2)^{2 s+1}-1}{(K+2)^{2 s}} \geqslant & \frac{(2 s+1) 2^{2 s+2}\left(1+2^{2 s}\right)}{2^{2 s}-1}=\frac{(2 s+1) 2^{2 s+2}\left(1+\frac{2^{2 s}-1}{2}\right)}{2^{2 s}-1-\frac{2^{2 s}-1}{2}} \\
& \geqslant \frac{(2 s+1) 2^{2 s+2}\left(1+2^{2 s+1} s(K+2)^{2 s} \vartheta_{\varepsilon}^{2 s}\left(\|\sigma\|_{\infty}+\hat{\delta}\right)\right)}{2^{2 s}-1-2^{2 s+1} s(K+2)^{2 s} \vartheta_{\varepsilon}^{2 s}\left(\|\sigma\|_{\infty}+\hat{\delta}\right)} \\
& \geqslant \frac{(2 s+1) 2^{2 s+2}\left(1+2 s(K+2)^{2 s} \vartheta_{\varepsilon}^{2 s}\|\sigma\|_{\infty}\right)}{2^{2 s}-1-2^{2 s+1} s(K+2)^{2 s} \vartheta_{\varepsilon}^{2 s}\left(\|\sigma\|_{\infty}+\hat{\delta}\right)}
\end{aligned}
$$

which implies

$$
\frac{s \vartheta_{\varepsilon}^{2 s+1}\left((K+2)^{2 s+1}-1\right)}{\gamma(2 s+1)\left(1+2 s(K+2)^{2 s} \vartheta_{\varepsilon}^{2 s}\|\sigma\|_{\infty}\right)} \geqslant \frac{2^{2 s+2} s(K+2)^{2 s} \vartheta_{\varepsilon}^{2 s+1}}{\gamma\left[2^{2 s}-1-2^{2 s+1} s(K+2)^{2 s} \vartheta_{\varepsilon}^{2 s}\left(\|\sigma\|_{\infty}+\hat{\delta}\right)\right]}=t_{\varepsilon} .
$$

The previous inequality and (5.48) give

$$
\tau \geqslant t_{\varepsilon}
$$

This inequality, (5.47), (5.26) and (5.35) imply (5.28). Finally, since $\hat{x}_{1}(t)$ is increasing by (5.50), (5.51) and (5.52) give (5.27). This completes the proof of Lemma 5.7.

\section{Proof of Theorem 1.4}

This section is devoted to the proof of Theorem 1.4. Let us consider the function

$$
h(t, x):=u\left(\frac{x-x(t)}{\varepsilon}\right)+\varrho_{\varepsilon} e^{-\frac{\mu t}{\varepsilon^{2 s}+1}}
$$

where

$$
x(t):=y_{\varepsilon}+K_{\varepsilon} \varrho_{\varepsilon}\left(e^{-\frac{\mu t}{\varepsilon^{2 s+1}}}-1\right),
$$

where $y_{\varepsilon}$ is given by Theorem 1.3 . We show that $h$ is a supersolution, as next result states:

Lemma 6.1. There exist $\varepsilon_{0}>0$ and $\mu>0$, such that for any $\varepsilon<\varepsilon_{0}$, there exists $K_{\varepsilon}=o(1)$ as $\varepsilon \rightarrow 0$ such that function $h$ defined in (6.1)-(6.2) satisfies

$$
\varepsilon h_{t}-\mathcal{I}_{s} h+\frac{1}{\varepsilon^{2 s}} W^{\prime}(h) \geqslant 0
$$

for any $x \in \mathbb{R}$ and $t>0$.

Proof. We compute

$$
\begin{aligned}
\varepsilon h_{t} & =-\dot{x} u^{\prime}\left(\frac{x-x(t)}{\varepsilon}\right)-\varepsilon^{-2 s} \varrho_{\varepsilon} \mu e^{-\frac{\mu t}{\varepsilon^{2 s+1}}} \\
& =\varepsilon^{-2 s-1} K_{\varepsilon} \varrho_{\varepsilon} \mu e^{-\frac{\mu t}{\varepsilon^{2 s+1}}} u^{\prime}\left(\frac{x-x(t)}{\varepsilon}\right)-\varepsilon^{-2 s} \varrho_{\varepsilon} \mu e^{-\frac{\mu t}{\varepsilon^{2 s+1}}},
\end{aligned}
$$

and

$$
\mathcal{I}_{s} h=\varepsilon^{-2 s} \mathcal{I}_{s} u\left(\frac{x-x(t)}{\varepsilon}\right)=\varepsilon^{-2 s} W^{\prime}\left(u\left(\frac{x-x(t)}{\varepsilon}\right)\right) .
$$


Then

$$
\begin{aligned}
\varepsilon h_{t}-\mathcal{I}_{s} h+\frac{1}{\varepsilon^{2 s}} W^{\prime}(h) & =\varepsilon^{-2 s-1} K_{\varepsilon} \varrho_{\varepsilon} \mu e^{-\frac{\mu t}{\varepsilon^{2 s+1}}} u^{\prime}\left(\frac{x-x(t)}{\varepsilon}\right)-\varepsilon^{-2 s} \varrho_{\varepsilon} \mu e^{-\frac{\mu t}{\varepsilon^{2 s+1}}} \\
& +\varepsilon^{-2 s} W^{\prime}\left(u\left(\frac{x-x(t)}{\varepsilon}\right)+\varrho_{\varepsilon} e^{-\frac{\mu t}{\varepsilon^{2 s+1}}}\right)-\varepsilon^{-2 s} W^{\prime}\left(u\left(\frac{x-x(t)}{\varepsilon}\right)\right) .
\end{aligned}
$$

Case 1. Suppose that $x$ is close to $x(t)$ more than $\kappa_{\varepsilon}$ :

$$
|x-x(t)| \leqslant \kappa_{\varepsilon}
$$

where $\kappa_{\varepsilon}$ is such that

$$
\frac{\varepsilon}{\kappa_{\varepsilon}}=o(1) \quad \text { as } \varepsilon \rightarrow 0
$$

Then estimate (2.2) implies

$$
u^{\prime}\left(\frac{x-x(t)}{\varepsilon}\right) \geqslant c\left(\frac{\varepsilon}{\kappa_{\varepsilon}}\right)^{1+2 s} .
$$

Moreover from the Lipschitz regularity of $W^{\prime}$ we get

$$
\varepsilon^{-2 s} W^{\prime}\left(u\left(\frac{x-x(t)}{\varepsilon}\right)+\varrho_{\varepsilon} e^{-\frac{\mu t}{\varepsilon^{2 s+1}}}\right)-\varepsilon^{-2 s} W^{\prime}\left(u\left(\frac{x-x(t)}{\varepsilon}\right)\right) \geqslant-\varepsilon^{-2 s} C \varrho_{\varepsilon} e^{-\frac{\mu t}{\varepsilon^{2 s+1}}} .
$$

Therefore, using in addition that $\varrho_{\varepsilon}=o(1)$ as $\varepsilon \rightarrow 0$, we get

$$
\begin{aligned}
\varepsilon h_{t}-\mathcal{I}_{s} h+\frac{1}{\varepsilon^{2 s}} W^{\prime}(h) \geqslant & \frac{K_{\varepsilon} \varrho_{\varepsilon} \mu}{\varepsilon^{2 s+1}} e^{-\frac{\mu t}{\varepsilon^{2 s+1}}} c\left(\frac{\varepsilon}{\kappa_{\varepsilon}}\right)^{1+2 s}-\frac{\varrho_{\varepsilon} \mu}{\varepsilon^{2 s}} e^{-\frac{\mu t}{\varepsilon^{2 s+1}}}-\frac{C \varrho_{\varepsilon}}{\varepsilon^{2 s}} e^{-\frac{\mu t}{\varepsilon^{2 s+1}}} \\
& =\varrho_{\varepsilon} e^{-\frac{\mu t}{\varepsilon^{2 s+1}}}\left(c K_{\varepsilon} \mu \kappa_{\varepsilon}^{-2 s-1}-\mu \varepsilon^{-2 s}-C \varepsilon^{-2 s}\right) \\
& =0
\end{aligned}
$$

if

$$
K_{\varepsilon} \mu=\frac{C+\mu}{c} \kappa_{\varepsilon}^{2 s+1} \varepsilon^{-2 s} .
$$

Case 2. Suppose that

$$
|x-x(t)| \geqslant \kappa_{\varepsilon},
$$

where $\kappa_{\varepsilon}$ satisfies (6.4). Then, 6.4), estimate (2.1) and

$$
W^{\prime \prime}(0)=\beta>0
$$

imply that for $\varepsilon$ small enough, we have

$$
W^{\prime \prime}\left(u\left(\frac{x-x(t)}{\varepsilon}\right)\right) \geqslant \frac{\beta}{2} .
$$


Therefore, we have

$$
\begin{aligned}
& W^{\prime}\left(u\left(\frac{x-x(t)}{\varepsilon}\right)+\varrho_{\varepsilon} e^{-\frac{\mu t}{\varepsilon^{2 s+1}}}\right)-W^{\prime}\left(u\left(\frac{x-x(t)}{\varepsilon}\right)\right)= \\
& W^{\prime \prime}\left(u\left(\frac{x-x(t)}{\varepsilon}\right)\right) \varrho_{\varepsilon} e^{-\frac{\mu t}{\varepsilon^{2 s+1}}}+O\left(\varrho_{\varepsilon} e^{-\frac{\mu t}{\varepsilon^{2 s+1}}}\right)^{2} \\
& \geqslant \frac{\beta}{2} \varrho_{\varepsilon} e^{-\frac{\mu t}{\varepsilon^{2 s+1}}}+O\left(\varrho_{\varepsilon} e^{-\frac{\mu t}{\varepsilon^{2 s+1}}}\right)^{2} \\
& \geqslant \frac{\beta}{4} \varrho_{\varepsilon} e^{-\frac{\mu t}{\varepsilon^{2 s+1}}},
\end{aligned}
$$

for $\varepsilon$ small enough. From 6.3 and the previous estimate, we conclude that

$$
\begin{aligned}
\varepsilon h_{t}-\mathcal{I}_{s} h+\frac{1}{\varepsilon^{2 s}} W^{\prime}(h) & \geqslant-\varepsilon^{-2 s} \varrho_{\varepsilon} \mu e^{-\frac{\mu t}{\varepsilon^{2 s}+1}}+\varepsilon^{-2 s} \frac{\beta}{4} \varrho_{\varepsilon} e^{-\frac{\mu t}{\varepsilon^{2 s+1}}} \\
& =\varepsilon^{-2 s} \varrho_{\varepsilon} e^{-\frac{\mu t}{\varepsilon^{2 s+1}}}\left(\frac{\beta}{4}-\mu\right) \geqslant 0
\end{aligned}
$$

if

$$
\mu \leqslant \frac{\beta}{4}
$$

The lemma is then proven choosing $\mu$ satisfying (6.6), $\kappa_{\varepsilon}$ satisfying (6.4) and the following

$$
\kappa_{\varepsilon}^{2 s+1} \varepsilon^{-2 s}=o(1) \quad \text { as } \varepsilon \rightarrow 0,
$$

and finally $K_{\varepsilon}$ satisfying 6.5$)$.

Let us now conclude the proof of Theorem 1.4. From Theorem 1.3 we have

$$
v_{\varepsilon}\left(T_{\varepsilon}^{1}, x\right) \leqslant h(0, x) \text { for any } x \in \mathbb{R} \text {. }
$$

Moreover, for $\mu$ and $K_{\varepsilon}=o(\varepsilon)$ as $\varepsilon \rightarrow 0$, given by Lemma 6.1 and $\varepsilon$ small enough, the function $h(t, x)$ is a supersolution of the equation (1.3). The comparison principle then implies

$$
v_{\varepsilon}\left(T_{\varepsilon}^{1}+t, x\right) \leqslant h(t, x) \text { for any } x \in \mathbb{R} \text { and } t>0,
$$

i.e. (1.18). Similarly we can prove inequality in 1.17 and this concludes the proof of the theorem.

\section{Proof of Corollary 1.5}

In order to complete the proof of Corollary 1.5, we follow the proof of Step 2 of Theorem 2 in [13], and we perform the necessary modifications needed in this case.

For fixed $\varepsilon$ the function $v_{\varepsilon}(t, x)$ is Hölder continuous in $x$ uniformly in time, see e.g. [10]. Then, there exists a sequence $\left(t_{k}\right)_{k}$ with $t_{k} \rightarrow+\infty$ as $k \rightarrow \infty$ such that

$$
v_{\varepsilon}\left(t_{k}, x\right) \rightarrow v_{\varepsilon}^{\infty}(x) \text { as } k \rightarrow \infty
$$

with $v_{\varepsilon}^{\infty}(x)$ viscosity solution of the stationary equation

$$
\mathcal{I}_{s} v=\frac{1}{\varepsilon^{2 s}} W^{\prime}(v) \quad \text { in } \mathbb{R}
$$


Under the assumptions (1.5), the function $v_{\varepsilon}^{\infty}$ is of class $C^{2, \alpha}(\mathbb{R})$ for some $\alpha$ depending of $s$, see for instance Lemma 5 in the Appendix of [13]. Moreover, for $\varepsilon$ small enough, by Theorem 1.4

$$
u\left(\frac{x-z_{\varepsilon}-K_{\varepsilon} \varrho_{\varepsilon}}{\varepsilon}\right) \leqslant v_{\varepsilon}^{\infty}(x) \leqslant u\left(\frac{x-y_{\varepsilon}+K_{\varepsilon} \varrho_{\varepsilon}}{\varepsilon}\right) \quad \text { for any } x \in \mathbb{R},
$$

where $u$ is the solution of 1.7 . Inequalities 7.1 and estimate 2.10 imply that

$$
\lim _{x \rightarrow-\infty} v_{\varepsilon}^{\infty}(x)=0 \text { and } \lim _{x \rightarrow+\infty} v_{\varepsilon}^{\infty}(x)=1 .
$$

Then there exists $x_{\varepsilon} \in \mathbb{R}$ such that $v_{\varepsilon}^{\infty}\left(x_{\varepsilon}\right)=\frac{1}{2}$. Let us denote

$$
u_{\varepsilon}(x):=u\left(\frac{x-x_{\varepsilon}}{\varepsilon}\right) \text {. }
$$

Remark that

$$
v_{\varepsilon}^{\infty}\left(x_{\varepsilon}\right)=u_{\varepsilon}\left(x_{\varepsilon}\right)=\frac{1}{2} .
$$

We want to show that

$$
v_{\varepsilon}^{\infty}(x)=u_{\varepsilon}(x) \text { for any } x \in \mathbb{R} .
$$

From (7.2), for any $0<a<1$ there exists $k(a) \in \mathbb{R}$ such that

$$
v_{\varepsilon}^{\infty}(x+k(a))+a>u_{\varepsilon}(x) \text { for any } x \in \mathbb{R} .
$$

Let us denote

$$
\bar{k}(a):=\inf \{k(a) \in \mathbb{R} \mid 7.6) \text { holds true }\} .
$$

Then, from $(7.2)$ and $a<1$, we have that $\bar{k}(a)$ is finite. Otherwise, choosing a minimizing sequence of $k(a)$ and passing to the limit along the sequence in (7.6), we would get a contradiction. The properties of the infimum imply that

$$
v_{\varepsilon}^{\infty}(x+k(a))+a>u_{\varepsilon}(x) \text { for any } x \in \mathbb{R}, k(a)>\bar{k}(a)
$$

and there exist sequences $\left(\eta_{j, a}\right)_{j},\left(x_{j, a}\right)_{j}$ with

$$
\eta_{j, a} \geqslant 0 \text { and } \lim _{j \rightarrow+\infty} \eta_{j, a}=0,
$$

such that

$$
v_{\varepsilon}^{\infty}\left(x_{j, a}+\bar{k}(a)-\eta_{j, a}\right)+a \leqslant u_{\varepsilon}\left(x_{j, a}\right) .
$$

We observe that $\left(x_{j, a}\right)_{j}$ must be bounded. Indeed, if

$$
\lim _{j \rightarrow+\infty} x_{j, a}= \pm \infty
$$

then we would have either

$$
a=\lim _{j \rightarrow+\infty} v_{\varepsilon}^{\infty}\left(x_{j, a}+\bar{k}(a)-\eta_{j, a}\right)+a \leqslant u_{\varepsilon}\left(x_{j, a}\right)=0,
$$

or

$$
1+a=\lim _{j \rightarrow+\infty} v_{\varepsilon}^{\infty}\left(x_{j, a}+\bar{k}(a)-\eta_{j, a}\right)+a \leqslant u_{\varepsilon}\left(x_{j, a}\right)=1,
$$

a contradiction. Therefore, we may suppose that

$$
\lim _{j \rightarrow+\infty} x_{j, a}=x_{a}
$$

for some $x_{a} \in \mathbb{R}$, and (7.7), (7.8) and the continuity of $v_{\varepsilon}^{\infty}$ and $u_{\varepsilon}$ imply 


$$
v_{\varepsilon}^{\infty}\left(x_{a}+\bar{k}(a)\right)+a=u_{\varepsilon}\left(x_{a}\right)
$$

and

$$
v_{\varepsilon}^{\infty}(x+\bar{k}(a))+a \geqslant u_{\varepsilon}(x), \quad \text { for any } x \in \mathbb{R} .
$$

Consequently

$$
\begin{aligned}
0 & \leqslant \int_{\mathbb{R}} \frac{v_{\varepsilon}^{\infty}(x+\bar{k}(a))+a-u_{\varepsilon}(x)}{\left|x-x_{a}\right|^{1+2 s}} d x \\
& =\mathcal{I}_{s} v_{\varepsilon}^{\infty}\left(x_{a}+\bar{k}(a)\right)-\mathcal{I}_{s} u_{\varepsilon}\left(x_{a}\right) \\
& =\varepsilon^{-2 s} W^{\prime}\left(v_{\varepsilon}^{\infty}\left(x_{a}+\bar{k}(a)\right)\right)-\varepsilon^{-2 s} W^{\prime}\left(u_{\varepsilon}\left(x_{a}\right)\right) \\
& =\varepsilon^{-2 s} W^{\prime}\left(u_{\varepsilon}\left(x_{a}\right)-a\right)-\varepsilon^{-2 s} W^{\prime}\left(u_{\varepsilon}\left(x_{a}\right)\right) .
\end{aligned}
$$

Now we claim that the sequence $\left(x_{a}\right)_{a}$ is bounded. Indeed, suppose that, up to subsequences,

$$
\lim _{a \rightarrow 0^{+}} x_{a}= \pm \infty
$$

then

$$
\text { either } \lim _{a \rightarrow 0^{+}} u_{\varepsilon}\left(x_{a}\right)=0 \text { or } \lim _{a \rightarrow 0^{+}} u_{\varepsilon}\left(x_{a}\right)=1 .
$$

Assumptions (1.5) on the potential imply that there exists $r>0$ such that

$$
W^{\prime}(u) \geqslant W^{\prime}(v)+\frac{\beta}{2}(u-v) \quad \text { if } u, v \in[0, r] \text { or } u, v \in[1-r, 1] \text { and } v \leqslant u,
$$

where $\beta=W^{\prime \prime}(0)>0$.

By (7.12) there exists $a_{0}>0$ such that both $u_{\varepsilon}\left(x_{a}\right)-a$ and $u_{\varepsilon}\left(x_{a}\right)$ belong to either $[0, r]$ or $[1-r, 1]$, for any $a \in\left(0, a_{0}\right)$. It follows that

$$
W^{\prime}\left(u_{\varepsilon}\left(x_{a}\right)-a\right)-W^{\prime}\left(u_{\varepsilon}\left(x_{a}\right)\right) \leqslant-\frac{\beta}{2} a
$$

and this is in contradiction with (7.11). Thus the sequence $\left(x_{a}\right)_{a}$ is bounded and we may suppose that, up to subsequences,

$$
\lim _{a \rightarrow 0^{+}} x_{a}=x_{0},
$$

for some $x_{0} \in \mathbb{R}$. We also have that the sequence $(\bar{k}(a))_{a}$ is bounded. Indeed, if

$$
\lim _{a \rightarrow 0^{+}} \bar{k}(a)= \pm \infty,
$$

we would obtain from (7.9) and (7.13) that, either

$$
0=\lim _{a \rightarrow 0^{+}} v_{\varepsilon}^{\infty}\left(x_{a}+\bar{k}(a)\right)=u_{\varepsilon}\left(x_{0}\right)
$$

or

$$
1=\lim _{a \rightarrow 0^{+}} v_{\varepsilon}^{\infty}\left(x_{a}+\bar{k}(a)\right)=u_{\varepsilon}\left(x_{0}\right)
$$

and this contradicts the fact that $0<u_{\varepsilon}(x)<1$ for any $x \in \mathbb{R}$. Thus $(\bar{k}(a))_{a}$ is bounded. Accordingly, we may suppose that

$$
\lim _{a \rightarrow 0^{+}} \bar{k}(a)=k_{0},
$$


for some $k_{0} \in \mathbb{R}$. Hence, passing to the limit as $a \rightarrow 0^{+}$in 7.11 , we conclude that

$$
P V \int_{\mathbb{R}} \frac{v_{\varepsilon}^{\infty}\left(x+k_{0}\right)-u_{\varepsilon}(x)}{\left|x-x_{0}\right|^{1+2 s}} d x=0 .
$$

On the other hand, by passing to the limit in $(7.10)$, we see that $v_{\varepsilon}^{\infty}\left(x+k_{0}\right)-u_{\varepsilon}(x) \geqslant 0$ for any $x \in \mathbb{R}$. We conclude that

$$
v_{\varepsilon}^{\infty}\left(x+k_{0}\right)=u_{\varepsilon}(x) \quad \text { for any } x \in \mathbb{R} .
$$

Recalling (7.4), we infer that $k_{0}=0$ and this gives (7.3). This completes the proof of Corollary 1.5 .

\section{Proof of the Results that are VAlid For both tWo AND three Particles}

In this section we prove the results which are auxiliary to the proofs of our main theorems and which are valid for both the cases of two and three particles. These results are Propositions 3.1, 5.1, 3.4, 5.3, and Lemmata 3.6, 5.6, 5.4 and 3.5. In what follows we will denote by $N$ the number of particles, then we may have either $N=2$ or $N=3$. We remark that the system of ODE's (3.2) can be written as (5.1) for $i=1,2$.

8.1. Proof of Propositions 3.1 and 5.1. In order to prove (5.4) and (3.5) suppose by contradiction that there is a sequence $\left(\delta_{k}\right)_{k}$, with $\delta_{k} \rightarrow 0$ as $k \rightarrow+\infty$ such that

$$
\lim _{k \rightarrow+\infty} T_{c}^{\delta_{k}}=T_{c}+2 a
$$

for some $a \in \mathbb{R} \backslash\{0\}$. Without loss of generality we may assume $a>0$. Then there exists $K$ such that for any $k>K$ the solution of system (5.1) with $\delta=\delta_{k}$ satisfies

$$
\min _{\substack{t \in\left[0, T_{c}+a\right] \\ i=1, \ldots, N-1}} \bar{x}_{i+1}(t)-\bar{x}_{i}(t) \geqslant M_{a}>0,
$$

for some $M_{a}$ independent of $k$. Accordingly the right-hand side of the equation in (5.1), together with its derivatives, is bounded when $t \in\left[0, T_{c}+a\right]$ by a quantity that depends on $a$. Therefore, we are in the position to apply the continuity result of the solution with respect to the parameter $\delta_{k}$. We obtain that, as $k \rightarrow+\infty$, the solution of (5.1) converges to $\left(x_{1}^{\infty}, \ldots, x_{N}^{\infty}\right)$, solution of 1.15$)$ in $\left[0, T_{c}+a\right]$ and satisfying (8.1). The continuity of $\left(x_{1}, \ldots, x_{N}\right)$ and $\left(x_{1}^{\infty}, \ldots, x_{N}^{\infty}\right)$ implies that there exists $\tau>0$ such that

$$
\begin{aligned}
m_{\tau}:=\min _{\substack{t \in\left[0, T_{c}-\tau\right] \\
i=1, \ldots, N}} x_{i+1}(t)-x_{i}(t) & \leqslant \min _{i=1, \ldots, N-1} x_{i+1}\left(T_{c}-2 \tau\right)-x_{i}\left(T_{c}-2 \tau\right) \\
& <M_{a} \\
& \leqslant \min _{i=1, \ldots, N-1} x_{i+1}^{\infty}\left(T_{c}-2 \tau\right)-x_{i}^{\infty}\left(T_{c}-2 \tau\right) .
\end{aligned}
$$

The right-hand side of the equation in 1.15 is Lipschitz continuous when $t \in\left[0, T_{c}-\tau\right]$ and $x_{i} \geqslant m_{\tau}$. Uniqueness results then imply that $x_{i}(t)=x_{i}^{\infty}(t)$ for any $t \in\left[0, T_{c}-\tau\right)$ and $i=1, \ldots, N$ which is in contradiction with 8.2 . This proves (5.4).

Next, from (5.4) we infer that for any $a>0$ we have

$$
\min _{\substack{t \in\left[0, T_{c}-a\right] \\ i=1, \ldots, N-1}} \bar{x}_{i+1}(t)-\bar{x}_{i}(t) \geqslant m_{a}>0
$$


with $m_{a}$ independent of $\delta$, and $(5.5)$ is then a consequence of continuity result of the solution of (5.1) with respect to the parameter $\delta$. With this, we have proved Propositions 3.1 and 5.1 .

8.2. Proof of Propositions 3.4 and 5.3. In order to simplify the notation, we set, for $i=1, \ldots, N$

$$
\tilde{u}_{i}(t, x):=u\left(\zeta_{i} \frac{x-\bar{x}_{i}(t)}{\varepsilon}\right)-H\left(\zeta_{i} \frac{x-\bar{x}_{i}(t)}{\varepsilon}\right),
$$

where $H$ is the Heaviside function and

$$
\psi_{i}(t, x):=\psi\left(\zeta_{i} \frac{x-\bar{x}_{i}(t)}{\varepsilon}\right) .
$$

Finally, let

$$
I_{\varepsilon}:=\varepsilon\left(\bar{v}_{\varepsilon}\right)_{t}+\frac{1}{\varepsilon^{2 s}} W^{\prime}\left(\bar{v}_{\varepsilon}\right)-\mathcal{I}_{s} \bar{v}_{\varepsilon}-\sigma .
$$

Roughly speaking, the quantity $I_{\varepsilon}$ denotes the error term in this equation (i.e., how far the modified dislocation $\bar{v}_{\varepsilon}$ is from being an exact solution). Thus, it is important to have careful estimates on this error term, as stated in the following result:

Lemma 8.1. For any $(t, x) \in\left(0, T_{c}^{\delta}\right) \times \mathbb{R}$ we have, for $i=1, \ldots, N$

$$
\begin{aligned}
I_{\varepsilon} & =O\left(\tilde{u}_{i}\right)\left(\varepsilon^{-2 s} \sum_{j \neq i} \tilde{u}_{j}+\bar{\sigma}+\zeta_{i} \bar{c}_{i} \eta\right)+\delta \\
& +\sum_{j=1}^{N}\left\{O\left(\varepsilon^{2 s+1} \dot{\bar{c}}_{j}\right)+O\left(\varepsilon^{2 s} \bar{c}_{j}^{2}\right)\right\} \\
& +\sum_{j \neq i}\left\{O\left(\bar{c}_{j} \psi_{j}\right)+O\left(\bar{c}_{j} \tilde{u}_{j}\right)+O\left(\varepsilon^{-2 s} \tilde{u}_{j}^{2}\right)\right\}+O\left(\varepsilon^{2 s}\right) .
\end{aligned}
$$

Proof. We have

$$
\begin{aligned}
\varepsilon\left(\bar{v}_{\varepsilon}\right)_{t} & =\varepsilon^{2 s+1} \bar{\sigma}_{t}-\sum_{j=1}^{N} \zeta_{j} \bar{c}_{j} u^{\prime}\left(\zeta_{j} \frac{x-\bar{x}_{j}}{\varepsilon}\right) \\
& +\sum_{j=1}^{N}\left(-\zeta_{j} \varepsilon^{2 s+1} \dot{\bar{c}}_{j} \psi\left(\zeta_{j} \frac{x-\bar{x}_{j}}{\varepsilon}\right)+\varepsilon^{2 s} \bar{c}_{j}^{2} \psi^{\prime}\left(\zeta_{j} \frac{x-\bar{x}_{j}}{\varepsilon}\right)\right) .
\end{aligned}
$$

Next, using the periodicity of $W$ and a Taylor expansion of $W^{\prime}$ at $\tilde{u}_{i}$, we compute:

$$
\begin{aligned}
\varepsilon^{-2 s} W^{\prime}\left(\bar{v}_{\varepsilon}\right) & =\varepsilon^{-2 s} W^{\prime}\left(\varepsilon^{2 s} \bar{\sigma}+\tilde{u}_{i}+\sum_{j \neq i} \tilde{u}_{j}-\zeta_{i} \varepsilon^{2 s} \bar{c}_{i} \psi_{i}-\sum_{j \neq i} \zeta_{j} \varepsilon^{2 s} \bar{c}_{j} \psi_{j}\right) \\
& =\varepsilon^{-2 s} W^{\prime}\left(\tilde{u}_{i}\right)+\varepsilon^{-2 s} W^{\prime \prime}\left(\tilde{u}_{i}\right)\left(\varepsilon^{2 s} \bar{\sigma}+\sum_{j \neq i} \tilde{u}_{j}-\zeta_{i} \varepsilon^{2 s} \bar{c}_{i} \psi_{i}-\sum_{j \neq i} \zeta_{j} \varepsilon^{2 s} \bar{c}_{j} \psi_{j}\right) \\
& +\sum_{j \neq i} O\left(\varepsilon^{-2 s} \tilde{u}_{j}^{2}\right)+\sum_{j=1}^{N} O\left(\varepsilon^{2 s} \bar{c}_{j}^{2} \psi_{j}^{2}\right)+O\left(\varepsilon^{2 s}\right)
\end{aligned}
$$


Finally, using (1.7) and (2.3), we evaluate

$$
\begin{aligned}
\mathcal{I}_{s} \bar{v}_{\varepsilon} & =\varepsilon^{2 s} \mathcal{I}_{s} \bar{\sigma}+\varepsilon^{-2 s} \mathcal{I}_{s} u\left(\zeta_{i} \frac{x-\bar{x}_{i}}{\varepsilon}\right)+\varepsilon^{-2 s} \sum_{j \neq i} \mathcal{I}_{s} u\left(\zeta_{j} \frac{x-\bar{x}_{j}}{\varepsilon}\right) \\
& -\zeta_{i} \bar{c}_{i} \mathcal{I}_{s} \psi\left(\frac{x-\bar{x}_{i}}{\varepsilon}\right)-\sum_{j \neq i} \zeta_{j} \bar{c}_{j} \mathcal{I}_{s} \psi\left(\zeta_{j} \frac{x-\bar{x}_{j}}{\varepsilon}\right) \\
& =O\left(\varepsilon^{2 s}\right)+\varepsilon^{-2 s} W^{\prime}\left(\tilde{u}_{i}\right)+\varepsilon^{-2 s} \sum_{j \neq i} W^{\prime}\left(\tilde{u}_{j}\right) \\
& -\zeta_{i} \bar{c}_{i}\left[W^{\prime \prime}\left(\tilde{u}_{i}\right) \psi_{i}+u^{\prime}\left(\zeta_{i} \frac{x-\bar{x}_{i}}{\varepsilon}\right)+\eta\left(W^{\prime \prime}\left(\tilde{u}_{i}\right)-W^{\prime \prime}(0)\right)\right] \\
& -\sum_{j \neq i} \zeta_{j} \bar{c}_{j}\left[W^{\prime \prime}\left(\tilde{u}_{j}\right) \psi_{j}+u^{\prime}\left(\zeta_{j} \frac{x-\bar{x}_{j}}{\varepsilon}\right)+\eta\left(W^{\prime \prime}\left(\tilde{u}_{j}\right)-W^{\prime \prime}(0)\right)\right] .
\end{aligned}
$$

Summing (8.6), 8.7) and (8.8), and noticing that the terms involving $u^{\prime}$, and the term

$$
\varepsilon^{-2 s} W^{\prime}\left(\tilde{u}_{i}\right)-\zeta_{i} \bar{c}_{i} W^{\prime \prime}\left(\tilde{u}_{i}\right) \psi_{i}
$$

appearing in both (8.7) and (8.8), cancel, we get

$$
\begin{aligned}
I_{\varepsilon} & =\varepsilon\left(\bar{v}_{\varepsilon}\right)_{t}+\varepsilon^{-2 s} W^{\prime}\left(\bar{v}_{\varepsilon}\right)-\mathcal{I}_{s} \bar{v}_{\varepsilon}-\sigma \\
& =\sum_{j=1}^{N}\left(-\zeta_{j} \varepsilon^{2 s+1} \dot{\bar{c}}_{j} \psi_{j}+\varepsilon^{2 s} \bar{c}_{j}^{2} \psi_{j}^{\prime}\right) \\
& -\varepsilon^{-2 s} \sum_{j \neq i} W^{\prime}\left(\tilde{u}_{j}\right)+W^{\prime \prime}\left(\tilde{u}_{i}\right)\left(\bar{\sigma}+\varepsilon^{-2 s} \sum_{j \neq i} \tilde{u}_{j}\right)+\sum_{j \neq i} \zeta_{j} \bar{c}_{j}\left(W^{\prime \prime}\left(\tilde{u}_{j}\right)-W^{\prime \prime}\left(\tilde{u}_{i}\right)\right) \psi_{j} \\
& +\zeta_{i} \bar{c}_{i} \eta\left(W^{\prime \prime}\left(\tilde{u}_{i}\right)-W^{\prime \prime}(0)\right)+\sum_{j \neq i} \zeta_{j} \bar{c}_{j} \eta\left(W^{\prime \prime}\left(\tilde{u}_{j}\right)-W^{\prime \prime}(0)\right)-\sigma \\
& +\sum_{j \neq i} O\left(\varepsilon^{-2 s} \tilde{u}_{j}^{2}\right)+\sum_{j=1}^{N} O\left(\varepsilon^{2 s} \bar{c}_{j}^{2} \psi_{j}^{2}\right)+O\left(\varepsilon^{2 s}\right) .
\end{aligned}
$$

Now, since $W^{\prime}(0)=0$, we use a Taylor expansion of $W^{\prime}$ around 0 , to see that

$$
\begin{aligned}
& -\varepsilon^{-2 s} \sum_{j \neq i} W^{\prime}\left(\tilde{u}_{j}\right)+W^{\prime \prime}\left(\tilde{u}_{i}\right)\left(\bar{\sigma}+\varepsilon^{-2 s} \sum_{j \neq i} \tilde{u}_{j}\right)+\zeta_{i} \bar{c}_{i} \eta\left(W^{\prime \prime}\left(\tilde{u}_{i}\right)-W^{\prime \prime}(0)\right) \\
& =-\varepsilon^{-2 s} \sum_{j \neq i} W^{\prime \prime}(0) \tilde{u}_{j}+W^{\prime \prime}\left(\tilde{u}_{i}\right)\left(\bar{\sigma}+\varepsilon^{-2 s} \sum_{j \neq i} \tilde{u}_{j}\right)+\zeta_{i} \bar{c}_{i} \eta\left(W^{\prime \prime}\left(\tilde{u}_{i}\right)-W^{\prime \prime}(0)\right)+\sum_{j \neq i} O\left(\varepsilon^{-2 s} \tilde{u}_{j}^{2}\right) \\
& =\varepsilon^{-2 s}\left(W^{\prime \prime}\left(\tilde{u}_{i}\right)-W^{\prime \prime}(0)\right) \sum_{j \neq i} \tilde{u}_{j}+W^{\prime \prime}\left(\tilde{u}_{i}\right) \bar{\sigma}+\zeta_{i} \bar{c}_{i} \eta\left(W^{\prime \prime}\left(\tilde{u}_{i}\right)-W^{\prime \prime}(0)\right)+\sum_{j \neq i} O\left(\varepsilon^{-2 s} \tilde{u}_{j}^{2}\right) \\
& =\left(W^{\prime \prime}\left(\tilde{u}_{i}\right)-W^{\prime \prime}(0)\right)\left(\varepsilon^{-2 s} \sum_{j \neq i} \tilde{u}_{j}+\bar{\sigma}+\zeta_{i} \bar{c}_{i} \eta\right)+W^{\prime \prime}(0) \bar{\sigma}+\sum_{j \neq i} O\left(\varepsilon^{-2 s} \tilde{u}_{j}^{2}\right),
\end{aligned}
$$


where we added and subtracted the term $W^{\prime \prime}(0) \bar{\sigma}$. Inserting 8.10 in $(8.9)$, we get

$$
\begin{aligned}
I_{\varepsilon} & =\left(W^{\prime \prime}\left(\tilde{u}_{i}\right)-W^{\prime \prime}(0)\right)\left(\varepsilon^{-2 s} \sum_{j \neq i} \tilde{u}_{j}+\bar{\sigma}+\zeta_{i} \bar{c}_{i} \eta\right)+W^{\prime \prime}(0) \bar{\sigma}-\sigma \\
& +\sum_{j=1}^{N}\left(-\zeta_{j} \varepsilon^{2 s+1} \dot{\bar{c}}_{j} \psi_{j}+\varepsilon^{2 s} \bar{c}_{j}^{2} \psi_{j}^{\prime}\right) \\
& +\sum_{j \neq i} \zeta_{j} \bar{c}_{j}\left(W^{\prime \prime}\left(\tilde{u}_{j}\right)-W^{\prime \prime}\left(\tilde{u}_{i}\right)\right) \psi_{j}+\sum_{j \neq i} \zeta_{j} \bar{c}_{j} \eta\left(W^{\prime \prime}\left(\tilde{u}_{j}\right)-W^{\prime \prime}(0)\right) \\
& +\sum_{j \neq i} O\left(\varepsilon^{-2 s} \tilde{u}_{j}^{2}\right)+\sum_{j=1}^{N} O\left(\varepsilon^{2 s} \bar{c}_{j}^{2} \psi_{j}^{2}\right)+O\left(\varepsilon^{2 s}\right) .
\end{aligned}
$$

Now from (5.7) it follows that

$$
W^{\prime \prime}(0) \bar{\sigma}-\sigma=\delta
$$

Moreover we have

$$
\begin{gathered}
\left(W^{\prime \prime}\left(\tilde{u}_{i}\right)-W^{\prime \prime}(0)\right)=O\left(\tilde{u}_{i}\right), \\
\varepsilon^{2 s} \bar{c}_{i}^{2} \psi_{i}^{\prime}, O\left(\varepsilon^{2 s} \bar{c}_{i}^{2} \psi_{i}^{2}\right)=O\left(\varepsilon^{2 s} \bar{c}_{i}^{2}\right), \\
\varepsilon^{2 s} \bar{c}_{j}^{2} \psi_{j}^{\prime}, O\left(\varepsilon^{2 s} \bar{c}_{j}^{2} \psi_{j}^{2}\right)=O\left(\varepsilon^{2 s} \bar{c}_{j}^{2}\right), \\
\bar{c}_{j}\left(W^{\prime \prime}\left(\tilde{u}_{j}\right)-W^{\prime \prime}\left(\tilde{u}_{i}\right)\right) \psi_{j}=O\left(\bar{c}_{j} \psi_{j}\right), \\
\bar{c}_{j} \eta\left(W^{\prime \prime}\left(\tilde{u}_{j}\right)-W^{\prime \prime}(0)\right)=O\left(\bar{c}_{j} \tilde{u}_{j}\right) .
\end{gathered}
$$

Equality (8.5) then follows.

Let us now conclude the proof of Propositions 3.4 and 5.3. Recalling (8.4), we want to find $\vartheta_{\varepsilon}$ such that for $\bar{x}_{i+1}(t)-\bar{x}_{i}(t) \geqslant \vartheta_{\varepsilon}, i=1, \ldots, N-1$, we have

$$
I_{\varepsilon}=o(1)+\delta \quad \text { as } \varepsilon \rightarrow 0 .
$$

Let us divide the proof in two cases.

Case 1. Suppose that $x$ is close to $\bar{x}_{i}(t)$ more than $\varepsilon^{\alpha}$, for some $i=1, \ldots, N$ :

$$
\left|x-\bar{x}_{i}(t)\right| \leqslant \varepsilon^{\alpha} \quad \text { with } 0<\alpha<\frac{\kappa-2 s}{\kappa},
$$

where $\kappa$ is given in Lemma 2.1. Let us assume that for $j \neq i$

$$
\left|\bar{x}_{j}(t)-\bar{x}_{i}(t)\right| \geqslant \vartheta_{\varepsilon}>2 \varepsilon^{\alpha}
$$

with $\vartheta_{\varepsilon}$ to be determined. Then for $j \neq i$

$$
\left|x-\bar{x}_{j}(t)\right| \geqslant\left|\bar{x}_{i}(t)-\bar{x}_{j}(t)\right|-\left|x-\bar{x}_{i}(t)\right| \geqslant\left|\bar{x}_{i}(t)-\bar{x}_{j}(t)\right|-\varepsilon^{\alpha} \geqslant \frac{\vartheta_{\varepsilon}}{2} .
$$


Hence, from (2.1) and (8.3), we get

$$
\begin{aligned}
& \left|\frac{\tilde{u}_{j}(t, x)}{\varepsilon^{2 s}}+\zeta_{j} \frac{1}{2 s W^{\prime \prime}(0)} \frac{x-\bar{x}_{j}(t)}{\left|x-\bar{x}_{j}(t)\right|^{1+2 s}}\right| \\
& =\frac{1}{\varepsilon^{2 s}}\left|u\left(\zeta_{j} \frac{x-\bar{x}_{j}(t)}{\varepsilon}\right)-H\left(\zeta_{j} \frac{x-\bar{x}_{j}(t)}{\varepsilon}\right)+\zeta_{j} \frac{\varepsilon^{2 s}}{2 s W^{\prime \prime}(0)} \frac{x-\bar{x}_{j}(t)}{\left|x-\bar{x}_{j}(t)\right|^{1+2 s}}\right| \\
& \leqslant C \frac{\varepsilon^{\kappa}}{\varepsilon^{2 s}} \frac{1}{\left|x-\bar{x}_{j}(t)\right|^{\kappa}} \\
& \leqslant C \varepsilon^{\kappa-2 s} \vartheta_{\varepsilon}^{-\kappa} .
\end{aligned}
$$

Next, a Taylor expansion of the function $\frac{x-\bar{x}_{j}(t)}{\left|x-\bar{x}_{j}(t)\right|^{1+2 s}}$ around $\bar{x}_{i}(t)$, gives

$$
\left|\frac{x-\bar{x}_{j}(t)}{\left|x-\bar{x}_{j}(t)\right|^{1+2 s}}-\frac{\bar{x}_{i}(t)-\bar{x}_{j}(t)}{\left|\bar{x}_{i}(t)-\bar{x}_{j}(t)\right|^{1+2 s}}\right| \leqslant \frac{2 s}{\left|\xi-\bar{x}_{j}(t)\right|^{1+2 s}}\left|x-\bar{x}_{i}(t)\right| \leqslant C \varepsilon^{\alpha} \vartheta_{\varepsilon}^{-(1+2 s)},
$$

where $\xi$ is a suitable point lying on the segment joining $x$ to $\bar{x}_{i}(t)$. The last two inequalities imply for $j \neq i$

$$
\left|\frac{\tilde{u}_{j}(t, x)}{\varepsilon^{2 s}}+\zeta_{j} \frac{1}{2 s W^{\prime \prime}(0)} \frac{\bar{x}_{i}(t)-\bar{x}_{j}(t)}{\left|\bar{x}_{i}(t)-\bar{x}_{j}(t)\right|^{1+2 s}}\right| \leqslant C\left(\varepsilon^{\kappa-2 s} \vartheta_{\varepsilon}^{-\kappa}+\varepsilon^{\alpha} \vartheta_{\varepsilon}^{-(1+2 s)}\right) .
$$

Therefore, from (8.5), we get that

$$
\begin{aligned}
I_{\varepsilon} & =O\left(\tilde{u}_{i}\right)\left(\sum_{j \neq i}-\zeta_{j} \frac{1}{2 s W^{\prime \prime}(0)} \frac{\bar{x}_{i}(t)-\bar{x}_{j}(t)}{\left|\bar{x}_{i}(t)-\bar{x}_{j}(t)\right|^{1+2 s}}+\bar{\sigma}+\zeta_{i} \bar{c}_{i} \eta\right)+\delta \\
& +O\left(\varepsilon^{\kappa-2 s} \vartheta_{\varepsilon}^{-k}+\varepsilon^{\alpha} \vartheta_{\varepsilon}^{-(1+2 s)}\right) \\
& +\sum_{j=1}^{N}\left\{O\left(\varepsilon^{2 s+1} \dot{\bar{c}}_{j}\right)+O\left(\varepsilon^{2 s} \bar{c}_{j}^{2}\right)\right\} \\
& +\sum_{j \neq i}\left\{O\left(\bar{c}_{j} \psi_{j}\right)+O\left(\bar{c}_{j} \tilde{u}_{j}\right)+O\left(\varepsilon^{-2 s} \tilde{u}_{j}^{2}\right)\right\}+O\left(\varepsilon^{2 s}\right) .
\end{aligned}
$$

Now, we compute the term between parenthesis in the first line above. From the definitions of $\bar{c}_{i}, \eta$ and $\bar{\sigma}$ given respectively in (5.6), (2.4) and (5.7), and the system of ODE's (5.1), we obtain

$$
\begin{aligned}
\sum_{j \neq i}-\zeta_{j} \frac{1}{2 s W^{\prime \prime}(0)} \frac{\bar{x}_{i}(t)-\bar{x}_{j}(t)}{\left|\bar{x}_{i}(t)-\bar{x}_{j}(t)\right|^{1+2 s}}+\bar{\sigma}+\zeta_{i} \bar{c}_{i} \eta & =\frac{\sigma(t, x)-\sigma\left(t, \bar{x}_{i}(t)\right)}{W^{\prime \prime}(0)} \\
& =O\left(\left|x-\bar{x}_{i}(t)\right|\right) \\
& =O\left(\varepsilon^{\alpha}\right) .
\end{aligned}
$$

Let us now estimate the remaining terms in (8.16). From (5.6), (5.1) and (8.13), we have for $j=1, \ldots, N$

$$
\left|\bar{c}_{j}\right|=O\left(\vartheta_{\varepsilon}^{-2 s}\right)
$$

then

$$
O\left(\varepsilon^{2 s} \bar{c}_{j}^{2}\right)=O\left(\varepsilon^{2 s} \vartheta_{\varepsilon}^{-4 s}\right)
$$


Next, differentiating (5.1) and using (5.6)

$$
\begin{aligned}
\dot{\bar{c}}_{i} & =\gamma \zeta_{i}\left(-\sum_{j \neq i} \zeta_{j} \frac{\dot{\bar{x}}_{i}-\dot{\bar{x}}_{j}}{\left|\bar{x}_{i}-\bar{x}_{j}\right|^{2 s+1}}-\sigma_{t}\left(t, \bar{x}_{i}(t)\right)-\sigma_{x}\left(t, \bar{x}_{i}(t)\right) \bar{c}_{i}\right) \\
& =-\gamma^{2} \zeta_{i} \sum_{j \neq i} \zeta_{j}\left|\bar{x}_{i}-\bar{x}_{j}\right|^{-2 s-1}\left(\sum_{k \neq i} \zeta_{i} \zeta_{k} \frac{\bar{x}_{i}-\bar{x}_{k}}{2 s\left|\bar{x}_{i}-\bar{x}_{k}\right|^{1+2 s}}\right. \\
& \left.-\zeta_{i} \sigma\left(t, \bar{x}_{i}\right)-\zeta_{i} \delta-\sum_{l \neq j} \zeta_{j} \zeta_{l} \frac{\bar{x}_{j}-\bar{x}_{l}}{2 s\left|\bar{x}_{j}-\bar{x}_{l}\right|^{1+2 s}}+\zeta_{j} \sigma\left(t, \bar{x}_{j}\right)+\zeta_{j} \delta\right) \\
& -\gamma \zeta_{i}\left(\sigma_{t}\left(t, \bar{x}_{i}(t)\right)+\sigma_{x}\left(t, \bar{x}_{i}(t)\right) \bar{c}_{i}\right) \\
& =O\left(\vartheta_{\varepsilon}^{-4 s-1}\right) .
\end{aligned}
$$

Then

$$
O\left(\varepsilon^{2 s+1} \dot{\bar{c}}_{j}\right)=O\left(\varepsilon^{2 s+1} \vartheta_{\varepsilon}^{-4 s-1}\right)=O\left(\varepsilon^{2 s} \vartheta_{\varepsilon}^{-4 s}\right),
$$

since $\varepsilon \vartheta_{\varepsilon}^{-1}=O\left(\varepsilon^{1-\alpha}\right)$ and $\alpha<1$.

Next, from (2.1) and (8.14), we have for $j \neq i$

$$
\left|\tilde{u}_{j}\right| \leqslant C \varepsilon^{2 s}\left|x-\bar{x}_{j}\right|^{-2 s} \leqslant C \varepsilon^{2 s} \vartheta_{\varepsilon}^{-2 s}
$$

then using (8.18), we get for $j \neq i$

$$
O\left(\bar{c}_{j} \tilde{u}_{j}\right)=O\left(\varepsilon^{2 s} \vartheta_{\varepsilon}^{-4 s}\right) .
$$

Similarly

$$
O\left(\varepsilon^{-2 s} \tilde{u}_{j}^{2}\right)=O\left(\varepsilon^{2 s} \vartheta_{\varepsilon}^{-4 s}\right) .
$$

Next, from 2.5 we know that for $|x| \geqslant \varepsilon^{\alpha-1}$

$$
|\psi(x)| \leqslant\left|\psi\left(\varepsilon^{\alpha-1}\right)\right|+C \varepsilon^{2 s(1-\alpha)} .
$$

Therefore, from 8.14 and 8.18 we get

$$
O\left(\bar{c}_{j} \psi_{j}\right)=O\left(\vartheta_{\varepsilon}^{-2 s} \psi\left(\varepsilon^{\alpha-1}\right)\right)+O\left(\varepsilon^{2 s(1-\alpha)} \vartheta_{\varepsilon}^{-2 s}\right) .
$$

Let us choose $\vartheta_{\varepsilon}$ such that

$$
\vartheta_{\varepsilon}, \varepsilon^{\alpha} \vartheta_{\varepsilon}^{-(1+2 s)}, \varepsilon^{2 s} \vartheta_{\varepsilon}^{-4 s}, \vartheta_{\varepsilon}^{-2 s} \psi\left(\varepsilon^{\alpha-1}\right), \varepsilon^{2 s(1-\alpha)} \vartheta_{\varepsilon}^{-2 s}=o(1) \quad \text { as } \varepsilon \rightarrow 0 .
$$

Remark that $\vartheta_{\varepsilon}>\varepsilon^{\alpha}$ implies $\varepsilon^{\kappa-2 s} \vartheta_{\varepsilon}^{-\kappa}<\varepsilon^{\kappa-2 s-\kappa \alpha}=o(1)$, since $\alpha$ satisfies the condition in (8.12). Then from (8.16), 8.17), 8.19), 8.20), 8.22), 8.23), 8.24) and 8.25) we obtain

$$
\begin{aligned}
I_{\varepsilon} & =O\left(\varepsilon^{\alpha}\right)+O\left(\varepsilon^{2 s} \vartheta_{\varepsilon}^{-4 s}\right)+O\left(\varepsilon^{\kappa-2 s} \vartheta_{\varepsilon}^{-\kappa}+\varepsilon^{\alpha} \vartheta_{\varepsilon}^{-(1+2 s)}\right) \\
& +O\left(\vartheta_{\varepsilon}^{-2 s} \psi\left(\varepsilon^{\alpha-1}\right)\right)+\varepsilon^{2 s(1-\alpha)} \vartheta_{\varepsilon}^{-2 s}+\delta \\
& =o(1)+\delta .
\end{aligned}
$$

Case 2. Suppose that for any $i=1, \ldots, N$ we have

$$
\left|x-\bar{x}_{i}(t)\right| \geqslant \varepsilon^{\alpha} .
$$


If for $j \neq i,\left|\bar{x}_{i}-\bar{x}_{j}\right| \geqslant \vartheta_{\varepsilon}$, with $\vartheta_{\varepsilon}>2 \varepsilon^{\alpha}$, we can assume that there exists $i$ such that for $j \neq i$

$$
\left|x-\bar{x}_{j}(t)\right| \geqslant \frac{\vartheta_{\varepsilon}}{2} .
$$

Then estimates 8.18), 8.19), 8.20, 8.21, 8.22, 8.23) and 8.24 hold. Moreover, using (2.1), we have

$$
\left|\tilde{u}_{i}\right| \leqslant C \varepsilon^{2 s}\left|x-\bar{x}_{i}\right|^{-2 s} \leqslant C \varepsilon^{2 s(1-\alpha)},
$$

and as a consequence, using in addition (8.21), for $j \neq i$

$$
O\left(\tilde{u}_{i}\right)\left(\varepsilon^{-2 s} \tilde{u}_{j}\right)=O\left(\varepsilon^{2 s(1-\alpha)} \vartheta_{\varepsilon}^{-2 s}\right) .
$$

Finally from (8.18), we have

$$
O\left(\tilde{u}_{i}\right) \bar{c}_{i}=O\left(\varepsilon^{2 s(1-\alpha)} \vartheta_{\varepsilon}^{-2 s}\right) .
$$

Then, if we assume (8.25), from 8.5), we obtain again 8.26).

We have proven (8.11). Now, we can choose $\delta=\delta_{\varepsilon}=o(1)$ as $\varepsilon \rightarrow 0$ such that

$$
I_{\varepsilon} \geqslant 0
$$

and the proposition is proven. With this, the statements in Propositions 3.4 and 5.3 are established.

8.3. Proof of Lemmata 3.6 and 5.6. In what follows, we will use the notation

$$
\bar{T}_{\varepsilon}^{1}:=T_{\varepsilon}^{1}
$$

when $N=2$. Let $\alpha$ be defined as in $(8.12)$ and $\vartheta_{\varepsilon}$ satisfying $(8.25)$. The monotonicity of $u$ implies that for $j=1, \ldots, N$

$$
u\left(\zeta_{j} \frac{x-\hat{x}_{j}(0)}{\varepsilon}\right) \geqslant u\left(\zeta_{j} \frac{x-\bar{x}_{j}^{\varepsilon}}{\varepsilon}\right) .
$$

We divide the proof in three cases. In the first two cases we will assume that the point $x$ is close enough to either $\bar{x}_{i}$ or $\hat{x}_{i}$ for some $i=1, \ldots, N$. This assumption will give a better estimate than (8.27), that will imply the desired result. In the third case, when $x$ is sufficiently far from all the particles, we will recover the result choosing conveniently $\hat{\delta} \geqslant \delta_{\varepsilon}$.

Case 1. Suppose that $x$ is close to $\bar{x}_{i}^{\varepsilon}$ more than $\varepsilon^{\alpha}$, for some $i=1, \ldots, N$ :

$$
\left|x-\bar{x}_{i}^{\varepsilon}\right| \leqslant \varepsilon^{\alpha} .
$$

Then, from estimate 2.1

$$
u\left(\zeta_{i} \frac{x-\bar{x}_{i}^{\varepsilon}}{\varepsilon}\right) \leqslant 1-C \varepsilon^{2 s} \varepsilon^{-2 s \alpha} .
$$

Next, from the initial conditions in (3.17) and in (5.21), we get

$$
\zeta_{i}\left(x-\hat{x}_{i}(0)\right)=\zeta_{i}\left(x-\bar{x}_{i}^{\varepsilon}\right)+\zeta_{i}\left(\bar{x}_{i}^{\varepsilon}-\hat{x}_{i}(0)\right) \geqslant-\varepsilon^{\alpha}+\vartheta_{\varepsilon} \geqslant \frac{\vartheta_{\varepsilon}}{2} .
$$

Therefore, from estimate 2.1

$$
u\left(\zeta_{i} \frac{x-\hat{x}_{i}(0)}{\varepsilon}\right) \geqslant 1-C \varepsilon^{2 s} \vartheta_{\varepsilon}^{-2 s} .
$$


Then, using in addition 8.27 we see that

$$
\begin{aligned}
\sum_{j=1}^{N}\left[u\left(\zeta_{j} \frac{x-\hat{x}_{j}(0)}{\varepsilon}\right)-u\left(\zeta_{j} \frac{x-\bar{x}_{j}^{\varepsilon}}{\varepsilon}\right)\right] & \geqslant u\left(\zeta_{i} \frac{x-\hat{x}_{i}(0)}{\varepsilon}\right)-u\left(\zeta_{i} \frac{x-\bar{x}_{i}^{\varepsilon}}{\varepsilon}\right) \\
& \geqslant C \varepsilon^{2 s} \varepsilon^{-2 s \alpha}-C \varepsilon^{2 s} \vartheta_{\varepsilon}^{-2 s} .
\end{aligned}
$$

Finally, remark that from (3.11), (5.9) and the initial conditions in (3.17) and in (5.21), for $i=1, \ldots, N$

$$
\bar{c}_{i}\left(\bar{T}_{\varepsilon}^{1}\right), \hat{c}(0)=O\left(\vartheta_{\varepsilon}^{-2 s}\right)
$$

We conclude that

$$
\hat{v}_{\varepsilon}(0, x)-\bar{v}\left(\bar{T}_{\varepsilon}^{1}, x\right) \geqslant C \varepsilon^{2 s} \varepsilon^{-2 s \alpha}-O\left(\varepsilon^{2 s} \vartheta_{\varepsilon}^{-2 s}\right)>0,
$$

for $\varepsilon$ small enough.

Case 2. Suppose that $x$ is close to $\hat{x}_{i}(0)$ more than $\varepsilon^{\alpha}$, for some $i=1, \ldots, N$.

$$
\left|x-\hat{x}_{i}(0)\right| \leqslant \varepsilon^{\alpha} \text {. }
$$

Then, from estimate 2.1

$$
u\left(\zeta_{i} \frac{x-\hat{x}_{i}}{\varepsilon}\right) \geqslant C \varepsilon^{2 s} \varepsilon^{-2 s \alpha} .
$$

Next, from the initial conditions in (3.17) and in (5.21), we get

$$
\zeta_{i}\left(x-\bar{x}_{i}^{\varepsilon}\right)=\zeta_{i}\left(x-\hat{x}_{i}(0)\right)+\zeta_{i}\left(\hat{x}_{i}(0)-\bar{x}_{i}^{\varepsilon}\right) \leqslant \varepsilon^{\alpha}-\vartheta_{\varepsilon} \leqslant-\frac{\vartheta_{\varepsilon}}{2} .
$$

Therefore, from estimate 2.1)

$$
u\left(\zeta_{i} \frac{x-\bar{x}_{i}^{\varepsilon}}{\varepsilon}\right) \leqslant C \varepsilon^{2 s} \vartheta_{\varepsilon}^{-2 s} .
$$

The conclusion then follows as in Case 1.

Case 3. Suppose that for any $i=1, \ldots, N$

$$
\left|x-\bar{x}_{i}^{\varepsilon}\right|,\left|x-\hat{x}_{i}(0)\right| \geqslant \varepsilon^{\alpha} .
$$

In this case, from 8.28 and 8.25 we have

$$
\bar{c}_{i}\left(\bar{T}_{\varepsilon}^{1}\right) \psi\left(\zeta_{i} \frac{x-\bar{x}_{i}^{\varepsilon}}{\varepsilon}\right), \hat{c}_{i}(0) \psi\left(\zeta_{i} \frac{x-\hat{x}_{i}(0)}{\varepsilon}\right)=o(1) .
$$

From the previous estimate and 8.27 , we get

$$
\hat{v}_{\varepsilon}(0, x)-\bar{v}\left(\bar{T}_{\varepsilon}^{1}, x\right) \geqslant \varepsilon^{2 s}\left(o(1)+\hat{\delta}-\delta_{\varepsilon}\right) .
$$

Therefore, we can choose $\hat{\delta}_{\varepsilon}=o(1)+\delta_{\varepsilon}$ such that

$$
\hat{v}_{\varepsilon}(0, x)-\bar{v}\left(\bar{T}_{\varepsilon}^{1}, x\right) \geqslant 0
$$

and this concludes the proof of the lemmata. These arguments establish Lemmata 3.6 and 5.6 .

8.4. Proof of Lemmata 5.4 and 3.5. The proof of Lemmata 5.4 and 3.5 is similar to the proof of Lemmata 3.6 and 5.6. For this reason we skip it. 


\section{REFERENCES}

[1] X. Cabré And Y. Sire, Nonlinear equations for fractional Laplacians II: existence, uniqueness, and qualitative properties of solutions, Trans. Amer. Math. Soc., 367 (2015) no. 2, 911-941.

[2] X. Cabré and J. SolÀ-Morales, Layer solutions in a half-space for boundary reactions, Comm. Pure Appl. Math., 58 (2005) no. 12, 1678-1732.

[3] S. Dipierro, A. Figalli and E. Valdinoci, Strongly nonlocal dislocation dynamics in crystals, Commun. Partial Differ. Equations 39 (2014) no. 12, 2351-2387.

[4] S. Dipierro, G. Palatucci and E. Valdinoci, Dislocation dynamics in crystals: a macroscopic theory in a fractional Laplace setting, Comm. Math. Phys., 333 (2015) no. 2, 1061-1105.

[5] E. Di Nezza, G. Palatucci and E. Valdinoci, Hitchhiker's guide to fractional Sobolev spaces, Bull. Sci. math., 136 (2012), no. 5, 521-573.

[6] A. Z. Fino, H. Ibrahim and R. Monneau, The Peierls-Nabarro model as a limit of a FrenkelKontorova model solutions in a half-space for boundary reactions, J. Differential Equations, 252 (2012), no. 1, 258-293.

[7] N. Forcadel, C. Imbert, R. Monneau, Homogenization of some particle systems with two-body interactions and of the dislocation dynamics, Discrete Contin. Dyn. Syst., 23 (2009), no. 3, 785-826.

[8] M. GonzÁlez And R. Monneau, Slow motion of particle systems as a limit of a reaction-diffusion equation with half-Laplacian in dimension one, Discrete Contin. Dyn. Syst., 32 (2012), no. 4, 12551286.

[9] R. L. Jerrard, Singular limits of scalar Ginzburg-Landau equations with multiple-well potentials, Adv. Differential Equations, 2 (1997), no. 1, 1-38.

[10] R. Monneau And S. Patrizi, Homogenization of the Peierls-Nabarro model for dislocation dynamics, J. Differential Equations, 253 (2012), no. 7, 2064-2015.

[11] F. R. N. Nabarro, Fifty-year study of the Peierls-Nabarro stress, Mat. Sci. Eng. A 234236 (1997), 67-76.

[12] T. OhtsukA, Motion of interfaces by an Allen-Cahn type equation with multiple-well potentials, Asymptot. Anal., 56 (2008), no. 2, 87-123.

[13] G. Palatucci, O. Savin and E. Valdinoci, Local and global minimizers for a variational energy involving a fractional norm. Ann. Mat. Pura Appl., (4) 192 (2013), 673-718.

[14] S. Patrizi and E. Valdinoci, Crystal dislocations with different orientations and collisions, Arch. Rational Mech. Anal., 217 (2015), 231-261.

[15] S. PAtrizi And E. VAldinoci, Homogenization and Orowan's law for anisotropic fractional operators of any order, Nonlinear Anal., Theory Methods Appl., Ser. A, Theory Methods, 119 (2015), 3-36.

[16] S. PATRIzi And E. VAldinoci, Long-time behavior for crystal dislocation dynamics, paper in progress.

[17] L. SilvestRe, Regularity of the obstacle problem for a fractional power of the Laplace operator, $\mathrm{PhD}$ thesis, University of Texas at Austin (2005), available online at http://math.uchicago.edu/ luis/preprints/luisdissreadable.pdf

(Stefania Patrizi and Enrico Valdinoci) Weierstrass Institut für Angewandte und Stochastik, Mohrenstrasse 39, D-10117 Berlin (Germany)

(Stefania Patrizi) Department Of Mathematics, University of Texas at Austin, 2515 Speedway, Austin TX 78712 (United States)

(Enrico Valdinoci) School of Mathematics and Statistics, University of Melbourne, 813 Swanston Street, Parkville ViC 3010 (Australia)

(Enrico Valdinoci) Dipartimento di Matematica Federigo EnRiques, Università Degli Studi Di Milano, Via Saldini 50, I-20133 Milano (Italy) 
(Enrico Valdinoci) Istituto di Matematica Applicata e Tecnologie Informatiche Enrico Magenes, Consiglio Nazionale delle Ricerche Via Ferrata 1, I-27100 Pavia (Italy)

E-mail address: Stefania.Patrizi@wias-berlin.de

E-mail address: Enrico.Valdinoci@wias-berlin.de 